\title{
Isometries of the hypercube: a tool for boolean regulatory networks analysis
}

\author{
Jean Fabre-Monplaisir, Brigitte Mossé and Élisabeth Remy
}

\begin{abstract}
Boolean finite dynamical systems (FDS) are commonly used in systems biology to model the dynamics of intracellular regulatory networks and interpret the emergence of cellular behaviors. Given a boolean FDS, we can compute the corresponding regulatory network, that is a directed signed graph representing all the interactions between components (genes), endowed with logical rules explaining the dynamical behaviour of the system. We consider the asynchronous trajectories generated by this boolean FDS and represent them on the hypercube. The exploration and analysis of this dynamics is a challenging task because of the combinatorial explosion that we face. A way to approach this problem is to exploit the links between the regulatory graph and the dynamics (FDS).

The set of isometries of the hypercube defines classes of boolean FDS gathering all the isometric FDS. Thus, we classify the set of boolean FDS on the basis of those isometries, and emphasize their common features through regulatory graphs and logical rules. We can then restrict the dynamical analysis of all the boolean FDS to one representative per class, and thereby considerably improve the efficiency of analysis of all the boolean FDS. Relying on invariants properties, we propose a constructive method to provide, given a FDS, a representative regulatory graph of its class.

We illustrate the efficiency of the method in concrete situations. For instance, the motif analysis $[12,13,3]$ is strongly improved thanks to the reduction of the space to explore. We also revisit the negative Thomas'rule $[14,15]$ by establishing a new demonstration.
\end{abstract}




\section{Contents}

1 Introduction $\quad 3$

2 Boolean finite dynamical systems and regulatory graphs 6

2.1 Boolean finite dynamical systems . . . . . . . . 6

2.2 Associated regulatory graphs . . . . . . . . . . . . 7

2.3 Logical framework . . . . . . . . . . . . . . . 8

3 Action of the symmetry group of the hypercube on boolean $\begin{array}{ll}\text { finite dynamical systems } & 9\end{array}$

3.1 The symmetry group of the hypercube . . . . . . . . . . 10

3.2 Action on boolean FDS and STG . . . . . . . . . . 10

3.3 Effect of a symmetry of the hypercube on regulatory graphs and logical rules . . . . . . . . . . . . . . . 12

3.3.1 Effect on local and global regulatory graphs . . . . . 13

3.3.2 Effect on paths and circuits . . . . . . . . . . . . 13

3.3.3 Effect on logical rules . . . . . . . . . . . . . . 14

3.4 A useful working tool . . . . . . . . . . . . . 18

4 Application to regulatory motifs analysis $\quad 19$

4.1 Isolated circuits . . . . . . . . . . . . . . . 20

4.2 Chorded circuits . . . . . . . . . . . . . . 21

4.3 Flower-graphs . . . . . . . . . . . . . . . . 23

4.3.1 Flower-graphs: study of $\phi_{f}(S) \ldots \ldots \ldots . . \ldots 25$

4.3.2 Flower-graphs: general case . . . . . . . . . . . 29

4.4 Extensions to hub-graphs . . . . . . . . . . . . . . 31

4.4.1 Hub-graphs: study of $\phi_{f}(S) \ldots \ldots \ldots \ldots$

4.4 .2 Hub-graphs: general case . . . . . . . . . . . 35

5 Application to the negative Thomas's rule 37

6 Conclusion $\quad 41$ 


\section{Introduction}

Finite dynamical systems are commonly used for the modelling of biological systems such as developmental, differentiation or homeostasis phenomena. The main objective is to explain and understand how and by whom these complex systems are controlled. Following a systems biology approach, we tackle this problem by studying the regulatory networks underlying the dynamical process.

Modelling of regulatory networks usually consists of gathering and synthetising the biological knowledge and data in two steps: first the construction of an interaction network representing the genes (nodes) and their regulations, activations or inhibitions (edges), and then its parameterisation coding the evolution of the systems induced by these interactions, that is called the dynamics. The panel of formalisms available to express the dynamics is wide, from quantitative to qualitative equations. Pioneer works in modelling of gene regulatory networks using boolean, and more generally discrete dynamical systems, go back to the 60 s with the works of S. Kaufman [7] and R. Thomas [20]. One of the strength of the discrete modelling is its ability to represent a wide range of dynamical behaviours while relying on a simple and easily handled mathematical formalism, not requiring quantitative parameters usually difficult to obtain with precision. Whatever the formalism used, the construction of a regulatory network and its parameterisation is a difficult task, with many unknowns and uncertainties on the processes and their mechanisms. Clearly, a priori good knowledge on the families of mathematical tools used to model the system may help and guide the modeller to overcome these difficulties. That is the aim of this theoretical work: improving the analysis of genetic regulatory networks, and making it more efficient.

This work focuses on the study of finite discrete systems (FDS) in the boolean case, a FDS representing the evolution of the system that has been modelised within the logical formalism. We aim to gather FDS displaying the same dynamical properties, and select a favorite class representative. For this purpose, we consider the hypercube, which supports the asynchronous boolean dynamics, and its symmetries; clearly, all the resulting FDS conserve the same properties. The study of this class of FDS allows to extract invariants relatively to their corresponding interaction graphs, and hence to have a deeper understanding of the links between the boolean dynamics and the structure of the underlying interaction graph. We adopt a geometrical

point of view that has already been used to study this classifying approach 
by the theoretical biologist and dynamicist Leon Glass in the 70's $[5,6]$.

An efficient application of this classification, together with a practical result allowing one to modify a regulatory graph while remaining in the class of symmetries, is emphasized through the analysis of regulatory motifs, which are known to biologists to play a major role in the structure of the trajectories space.

\section{Sections summary}

Notice Proofs and results presented in this paper go deeply into mathematical details, with geometrical and combinatorics arguments and tools developed in a series of propositions and remarks, allowing a fine understanding of some dynamical features. The reader may choose the level of reading; it is possible to capture the main results stated in the propositions and theorems without going into all details.

In Section 3, we give basics on boolean networks and logical formalism $[17,20]$.

Starting from a boolean finite dynamical system (FDS), that is a map $S$ from $X=\{0,1\}^{n}$ to itself ( $n$ is a fixed integer), we associate to it dynamics. The set $X$ of boolean vectors is the set of states, and trajectories are represented through state transition graphs (STG). The function $S$ encodes the dynamics, and updating rules are precised to describe the trajectories. The usual rules considered are the synchronous one, with trajectories given by the iteration of $S$, and the asynchronous one, which is non deterministic since only one component can update at a time and thus a state may have several successors. Two types of attractors may be obtained in these dynamics, complex attractors or stable states. Given $S$, we derive the corresponding regulatory graph $(\mathrm{RG})$, consisting in all activations and inhibitions between components $g_{1}, \ldots, g_{n}$ (usually genes in biological applications).

The logical formalism provides an other way to determine $S$, starting from a regulatory graph with $n$ vertices $g_{1}, \ldots, g_{n}$ and logical rules giving a complete description of the action of the regulators of each component; these rules allow enable the reconstitution of $S$.

In Section 4, we introduce the symmetry group $\Gamma_{n}$ of the hypercube of the $\mathrm{n}$ dimensional space $\mathbb{R}^{n}$. From a geometrical point of view, $\Gamma_{n}$ acts naturally on boolean asynchronous STG, when they are embedded in $\mathbb{R}^{n}$. We precise how an element $f$ of $\Gamma_{n}$ acts on a boolean FDS and on its synchronous and asynchronous STG. 
The action of $\Gamma_{n}$ on boolean FDS was first introduced by L.Glass [5] for the classification of asynchronous STG.In [22, 2], this action is studied with symbolical considerations, and the authors note that isometric STG are also isomorphic (in the sense of graph theory, renumbering of the nodes preserving adjacency). In Proposition 2 we emphasize a class of dynamics for which isomorphic STG are also isometric.

We also detail the transformation of the RG under a given symmetry (the topology and the signs of the circuits are conserved, but signs in paths may change), and precise the effect on logical rules.

We end this section with Proposition 7, a key tool to use these results in applications. It provides a way to transform a boolean STG $S$ in new ones, representatives of interest of the class of $S$ under the action of $\Gamma_{n}$ (by identifying subgraphs of the $R G$, on which it is possible to choose the signs of the interactions after the action of some symmetry).

In Section 5, we focus on the study of boolean FDS whose RG are usual regulatory motifs in biological applications: isolated circuits, chorded circuits, flower-graphs, and more generally hub-graphs. All these motifs are connected graphs containing at most one component with more than one regulator (the "hub"). The aim is to describe the dynamical behaviours that can be generated by a given regulatory motif, and eventually associate dynamical properties to this motif. Due to the large set of motif-compatible dynamics, this exhaustive and general study is difficult to carry out, and we show that the results of Section 4 greatly facilitate the study. For each motif, we adopt the same strategy: using Proposition 7, we transform any boolean FDS whose RG is the motif into an isometric one, whose RG has only activations all along a chosen sub-graph (activations are mathematically easier to study in the boolean dynamics, as they propagate coordinates along trajectories). Relying on invariants observed in Section 4, we deduce the dynamics associated to the motif through the analysis of the chosen isometrical dynamics. In particular, we provide a description of the attractors of the asynchronous dynamics. We find results already described in $[13,11]$ for the isolated and chorded circuits, and provide new ones related to flower-graphs and hub-graphs (Theorems 1,2 and 3). This analysis allows us to precise the number of classes of isometric FDS determined by the simplest motifs (isolated circuits, chorded circuits, flower-graphs with two petals). In the large family of hub-graphs, this analysis shows that only three asymptotic asynchronous behaviors may occur, depending on the way the regulators "cooperate" to regulate the hub (see Propositions 12 and 15, and Remarks 6 and 10), improving results of [3]. 
Finally, in Section 6, we use Proposition 7 to propose a new proof of the so-called "negative Thomas's rule" : Given $S: X \rightarrow X$ a boolean FDS such that its asynchronous dynamics displays a cyclic attractor, then the associated regulatory graph $\mathcal{R G}(S)$ contains a negative circuit [14, 15]. Indeed, Theorem 4 states that if $\mathcal{R G}(S)$ contains only positive circuits, then the asynchronous dynamics cannot display any cyclic attractors.

\section{Boolean finite dynamical systems and regula- tory graphs}

In the following, we consider a $n$ dimensions hypercube $\mathcal{H}_{n}$, that is the convex polyhedra of the space $\mathbb{R}^{n}$ whose set of vertices is equal to $X=\{0,1\}^{n}$; the elements $x=\left(x_{1}, \ldots, x_{n}\right)$ of $X$ will also be called states.

Notations 1. - For $a \in\{0,1\}, \bar{a}=1-a$.

- For $a \in\{0,1\}$ and $\alpha \in\{ \pm 1\}, a^{\alpha}=\left\{\begin{array}{ll}a & \text {, if } \alpha=+1 \\ \bar{a} & \text {, if } \alpha=-1\end{array}\right.$.

- For $x \in X, \bar{x}=\left(\overline{x_{1}}, \ldots, \overline{x_{n}}\right)$ and $\bar{x}^{j}=\left(x_{1}, \ldots, x_{j-1}, \overline{x_{j}}, x_{j+1}, \ldots, x_{n}\right)$.

- For $x \in X$, and $J \subset\{1, \ldots, n\}, \bar{x}^{J}=\left(y_{1}, \ldots, y_{n}\right)$, where $y_{i}=\left\{\begin{array}{ll}x_{i} & , \text { if } i \in J \\ \overline{x_{i}} & , \text { if } i \notin J\end{array}\right.$.

Notations 2. Thereafter, we will consider different directed graphs, and if $n$ and $n^{\prime}$ are nodes of a considered graph, we will write $n \rightsquigarrow n^{\prime}$ to indicate that there is a path from $n$ towards $n^{\prime}$ in this graph.

\subsection{Boolean finite dynamical systems}

We proceed from a boolean finite dynamical system $S$ (FDS, [17]), that is a transformation $S: X \rightarrow X$. Given the transformation $S=\left(S_{1}, \ldots, S_{n}\right)$, to any state $x$ is associated its updating set

$$
U p d_{S}(x)=\left\{i \in\{1, \ldots n\} ; S_{i}(x) \neq x_{i}\right\} .
$$

This brings to consider two usual dynamics associated to $S$ [4]:

- The synchronous dynamics, with a simultaneous change of all the coordinates of the state $x$ specified by $U p d_{S}(x)$. Any state has exactly one successor, its image under $S$, and this dynamics is deterministic. The graph of 
$S$, denoted $\mathcal{G}_{s}(S)$, is called synchronous state transition graph (synchronous STG) or synchronous graph. This graph gives the iteration of $S$ and thus the trajectories of the dynamics.

- The asynchronous dynamics, where at most one coordinate changes at a time. Hence, a state $x$ has as many successors as the cardinal of $U p d_{S}(x)$, and this dynamics is non-deterministic. We represent the asynchronous dynamics by a directed graph $\mathcal{G}_{a}(S)$, called asynchronous state transition graph (asynchronous STG) or asynchronous graph; the set of nodes of $\mathcal{G}_{a}(S)$ is the set $X$ of the states, and the edges, linking two consecutive states in the dynamics, are located on the edges of the hypercube $\mathcal{H}_{n}$. The trajectories are the paths of $\mathcal{G}_{a}(S)$, along edges of $\mathcal{H}_{n}$.

Let us remark that $\mathcal{G}_{a}(S)$ can be directly constructed from $\mathcal{G}_{s}(S)$, and conversely.

Our attention turns to attractors of these dynamics, which can be of two kinds: stable states (fixed points), and "cyclic" attractors (terminal strongly connected components of the considered STG). Let us remark that the stable states are the same in the synchronous and the asynchronous dynamics.

\section{$2.2 \quad$ Associated regulatory graphs}

A regulatory graph $(\mathrm{RG})$ is classically associated to the FDS. Let us denote this graph by $\mathcal{R G}(S)$. The $n$ vertices $g_{1}, \ldots, g_{n}$ of $\mathcal{R G}(S)$ are components whose levels are given by the $x_{i}$ along the dynamics. There is an edge in $\mathcal{R G}(S)$ from $g_{i}$ to $g_{j}$, called interaction from $g_{i}$ to $g_{j}$, if there exists two states $x$ and $y=\bar{x}^{i}$ such that $S_{j}(x) \neq S_{j}(y)$. This condition means that $g_{i}$ is a regulator of $g_{j}$, in the sense that the level $x_{i}$ of $g_{i}$ has an influence on the next level $x_{j}$ of $g_{j}$ under the action of $S$, expressed on at least one pair of states $\left\{x, \bar{x}^{i}\right\}$ of the space $X$.

It is therefore possible to build the graph $\mathcal{R G}(S)$ by superposing for all the states $x$ the local graphs, denoted by $\mathcal{R} \mathcal{G}_{x}(S)$, whose vertices are the $g_{i}$ and edges are the edges of $\mathcal{R G}(S)$ expressed at the pairs of states $\left\{x, \bar{x}^{i}\right\}$, for $i=1, \ldots, n$. Given a state $x$, it is natural to provide a sign to each interaction from $g_{i}$ to $g_{j}$ of $\mathcal{R} \mathcal{G}_{x}(S)$ : this sign is +1 (activation) if $\left(x_{i}-\bar{x}_{i}^{i}\right)\left(S_{j}(x)-S_{j}\left(\bar{x}^{i}\right)\right)>0$ and -1 (inhibition) otherwise. Then we will give a sign to an interaction of $\mathcal{R G}(S)$ in those cases where its sign is the same in all the local graphs where it is expressed. Considering a path in a $\mathrm{RG}$ or in some union of local RG, the sign of the path is the product of the signs of its interactions, when these signs are determined (in other words the same in the local graphs where expressed).

Without the loss of generality, we work with transformations $S$ such that 
$\mathcal{R G}(S)$ is connected; otherwise, the study of the dynamics returns to the study of the dynamics associated to each connected component of $\mathcal{R} \mathcal{G}(S)$.

As an illustration, we give a first proposition that we will use in several applications (cf. Section 4):

Proposition 1. Let $S$ be a boolean FDS on $X$, and $g_{1}, \ldots, g_{n}$ the related components. If a component $g_{j}$ has a unique regulator $g_{i}$, then for each state $x$, the interaction from $g_{i}$ to $g_{j}$ is expressed in the local graph $\mathcal{R} \mathcal{G}_{x}(S)$, and always with the same sign.

Proof. Let $x$ be a state such that $x_{i}=0$ and the interaction from $g_{i}$ to $g_{j}$ is expressed in the local graph $\mathcal{R G}_{x}(S)$ with the sign $\alpha$ (and thus also expressed in $\mathcal{R G}_{\bar{x}^{i}}(S)$ with the same sign), that is $S_{j}(x)=0^{\alpha}$ and $S\left(\bar{x}^{i}\right)_{j}=1^{\alpha}$.

For $k \in\{1, \ldots, n\} \backslash\{i\}$, let us consider the state $y=\bar{x}^{k}$. We must have $S_{j}(y)=0^{\alpha}$ and $S_{j}\left(\bar{y}^{i}\right)=1^{\alpha}$, for otherwise there is an interaction from $g_{k}$ to $g_{j}$. Therefore, the interaction from $g_{i}$ to $g_{j}$ is expressed in the local graphs $\mathcal{R G}_{y}(S)$ and $\mathcal{R G}_{\bar{y}^{i}}(S)$ with the sign $\alpha$.

The iteration of the move from a state $x$ to a state $y$, and the different possible choices of $k$ in $\{1, \ldots, n\} \backslash\{i\}$ allow to cover all the space $X$, and this gives the conclusion.

\subsection{Logical framework}

Another way to determine $S$ is to use the so called logical formalism [20], starting from a RG with $n$ vertices $g_{1}, \ldots, g_{n}$ and "logical rules" or "formulas": for each $g_{i}$, conditions on the levels of its regulators are assigned in a disjunctive normal form, that determine the states for which the level of $g_{i}$ becomes equal to 1 under $S$, that is the set $S_{i}^{-1}(1)$.

More precisely, we call radical any $g_{i}$ (meaning that the level of $g_{i}$ is equal to 1 ) or $\neg g_{i}$ (meaning that the level of $g_{i}$ is equal to 0 ), and conjunctive clause any conjunction of radicals; a disjunctive normal form is a disjunction of such clauses. The truth table of a clause (resp. a logical rule) is evaluated on the set $X$ of states, and a particular state $x$ makes it true if its value on $x$ is equal to 1 . In the sequel, we consider that clauses (resp. logical rules) having the same truth table are equal.

The following remarks, although immediate or classical, should be able to familiarize the reader to logical rules.

Remark 1. 1. To compute disjunctive logical rules from $S$, one can consider brutally for each $i \in\{1, \ldots, n\}$ all the states $x$ that lead to 
$S_{i}(x)=1$. Then, $\nu$ standing for the empty word, a correct logical rule for $g_{i}$ is

$$
C\left(g_{i}\right)=\bigvee_{x \in S_{i}^{-1}(1)} c(x)
$$

where, for $x=\left(x_{1}, \ldots, x_{n}\right) \in X$, the conjunctive clause $c(x)$ is defined by $c(x)=\bigwedge_{k \in\{1, \ldots, n\}} w_{k} g_{k}$ and $w_{k}=\left\{\begin{array}{ll}\nu & , \text { if } x_{k}=1 \\ \neg & , \text { if } x_{k}=0\end{array}\right.$.

2. A shortest logical rule for $g_{i}$ does not include any component that is not a regulator of $g_{i}$, and any logical rule for $g_{i}$ includes all its regulators.

3. For instance, in the particular case where a component $g_{i}$ has only activators, it admits a logical rule of the form

$$
C\left(g_{i}\right)=\bigvee_{j \in\{1, \ldots, s\}} \bigwedge_{k \in\left\{1, \ldots, r_{j}\right\}} g_{i_{j, k}},
$$

where the components $g_{i_{j, k}}$ are activators of $g_{i}$.

\section{Action of the symmetry group of the hypercube on boolean finite dynamical systems}

In order to give a structural classification of the boolean asynchronous STG for a fixed value of $n$, we are going to consider these graphs embedded in the $\mathrm{n}$ dimensional euclidian space $\mathbb{R}^{n}$ : the set $X=\{0,1\}^{n}$ of their vertices is naturally included in $\mathbb{R}^{n}$, and is also the set of the vertices of the $n$ dimensional hypercube $\mathcal{H}_{n}=[0,1]^{n}$.

Given $S$ a boolean FDS on $X$, we locate the edges of the asynchronous STG of $S$ on the edges of $\mathcal{H}_{n}$. More precisely, let $\left(\vec{e}_{i}\right)_{1 \leq i \leq n}$ be the usual basis of $\mathbb{R}^{n}$, given by $\overrightarrow{e_{i}}=(0, \ldots, 0,1,0, \ldots, 0)$ with " 1 " at the $i$ th place. We represent at each point $x \in X$ on edges of $\mathcal{H}_{n}$ the non zero components of $\overrightarrow{x S(x)}$ with respect to this basis (loops are not represented).

Let us remark that the synchronous STG of $S$ may also be embedded in $\mathbb{R}^{n}$, on diagonals of $\mathcal{H}_{n}$, by representing at each point $x \in X$ the vector $\overrightarrow{x S(x)}$ when it is non zero.

In this geometrical perspective, two asynchronous STG have the same structure if they can be superimposed under the action of an isometry of $\mathcal{H}_{n}=[0,1]^{n}$. This point of view is not new and was first introduced by L.Glass [6]. It has been taken up in recent works $[22,2]$. 


\subsection{The symmetry group of the hypercube}

The set of the isometries of $\mathbb{R}^{n}$ that fix $\mathcal{H}_{n}$ (or, in an equivalent manner, that fix $X)$ is a well-known group called symmetry group of $\mathcal{H}_{n}[18]$; let $\Gamma\left(\mathcal{H}_{n}\right)$ denote this group. From a purely algebraic point of view, $\Gamma\left(\mathcal{H}_{n}\right)$ is isomorphic to the semidirect product of the group $\mathbb{Z} / 2 \mathbb{Z}^{n}$ and the symmetric group $S_{n}$ of permutations of a set with $n$ elements [9]. The geometrical interpretation is that these isometries are one-to-one transformations composed of a permutation of the axis and of hyperplane symmetries exchanging parallel hyperfaces of $\mathcal{H}_{n}$. The effect of the permutation on a state $x=\left(x_{1}, \ldots, x_{n}\right)$ is the corresponding permutation of its coordinates, whereas each hyperplane symmetry transforms $x=\left(x_{1}, \ldots, x_{n}\right)$ into $\bar{x}^{j}=\left(x_{1}, \ldots, x_{j-1}, \overline{x_{j}}, x_{j+1}, \ldots, x_{n}\right)$ for some fixed element $j$ of $\{1, \ldots, n\}$. Let us remark that it is classical to call the elements $f$ of $\Gamma\left(\mathcal{H}_{n}\right)$ "symmetries", even if $f$ is not a symmetry in the usual sense (that is even if $f \circ f(M) \neq M$ for some points $M$ ).

In the following, we are opting for a geometrical point of view, since isometries of $\mathcal{H}_{n}$ will act naturally on various geometrical objects that we will consider. To this end, let us recall that to any isometry $f$ of the space $\mathbb{R}^{n}$ is associated a linear transformation $\vec{f}$ preserving orthogonality, such that the equality $f(M+\vec{u})=f(M)+\vec{f}(\vec{u})$ holds for any point $M$ and vector $\vec{u}$ of the space. If $f$ belongs to $\Gamma\left(\mathcal{H}_{n}\right)$, it is completely determined by $\vec{f}$, due to the fact that the center of $\mathcal{H}_{n}$ is a fixed point of $f$.

The previous description of $\Gamma\left(\mathcal{H}_{n}\right)$ shows that there exists a permutation $\sigma$ of $\{1, \ldots, n\}$ and $\left(\varepsilon_{1}, \ldots, \varepsilon_{n}\right) \in\{-1,+1\}^{n}$ such that $\vec{f}\left(\overrightarrow{e_{i}}\right)=\varepsilon_{\sigma(i)} \overrightarrow{e_{\sigma(i)}}$, and for $x=\left(x_{1}, \ldots, x_{n}\right) \in X$, the equality $f(x)=\left(x_{\sigma^{-1}(1)}^{\varepsilon_{1}}, \ldots, x_{\sigma^{-1}(n)}^{\varepsilon_{n}}\right)$ holds.

\subsection{Action on boolean FDS and STG}

Given $S: X \rightarrow X$ a boolean FDS and $f$ in the group $\Gamma\left(\mathcal{H}_{n}\right)$, we define a new "conjugated" FDS, $\phi_{f}(S)=f \circ S \circ f^{-1}$, where, for simplicity of notation, we continue to write $f$ for the restriction of $f$ to $X$. This defines an action of the group $\Gamma\left(\mathcal{H}_{n}\right)$ on the set of all the boolean FDS on $X$ (see [9] for more details) and gives a way of classification of these FDS: two boolean FDS are in the same class if there exists an element of $\Gamma\left(\mathcal{H}_{n}\right)$ sending one to the other. In this way we obtain what we call isometric boolean FDS, and isometric STG. Combinatorial tools related to group actions on finite sets and configurations have already been used to study this classifying approach $[5,6]$.

Once given $S$ and $f$, it is natural to compare the synchronous and asynchronous STG of $S$ and $\phi_{f}(S)$. Let us recall that two directed graphs are 


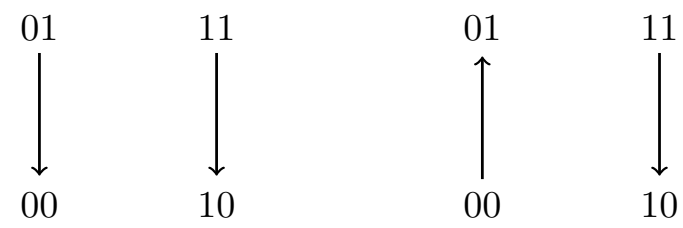

Figure 1: Two isomorphic but not isometric STG

isomorphic if there exists a one-to-one correspondence between their sets of nodes preserving adjacency (here in a directed meaning).

It is clear that the asynchronous graph $\mathcal{G}_{a}\left(\phi_{f}(S)\right)$ of $\phi_{f}(S)$ is the image of $\mathcal{G}_{a}(S)$ under $f$, from the fact that the nodes and the edges of $\mathcal{G}_{a}(S)$ ) are located on the vertices and some edges of $\mathcal{H}_{n}$. Therefore the isometric STG $\mathcal{G}_{a}\left(\phi_{f}(S)\right)$ and $\left.\mathcal{G}_{a}(S)\right)$ are isomorphic, the isometry $f$ carrying out the isomorphism. Consequently, the isometric synchronous STG $\mathcal{G}_{s}\left(\phi_{f}(S)\right)$ and $\mathcal{G}_{s}(S)$ ), easily geometrically constructed from the asynchronous ones, are also isomorphic.

Figure 1 represents a very simple example in dimension 2 of isomorphic but non isometric asynchronous graphs proposed in [21] (page 19).

Conversely, let us mention a current situation where isomorphic asynchronous graphs are necessarily isometric.

Proposition 2. Let $S_{1}$ and $S_{2}$ be two boolean FDS such that the asynchronous graph $\mathcal{G}_{a}\left(S_{1}\right)$ involves all the edges of $\mathcal{H}_{n}$. If $\mathcal{G}_{a}\left(S_{1}\right)$ and $\mathcal{G}_{a}\left(S_{2}\right)$ are isomorphic, then they are isometric.

Proof. We consider $n \geq 2$, the proposition being trivial for $n=1$, and we suppose the existence of an isomorphism from $\mathcal{G}_{a}\left(S_{1}\right)$ to $\mathcal{G}_{a}\left(S_{2}\right)$, i.e. of a one-to-one correspondence $\phi: X \rightarrow X$ associating nodes of $\mathcal{G}_{a}\left(S_{1}\right)$ to nodes of $\mathcal{G}_{a}\left(S_{2}\right)$ and preserving directed adjacency. Then the hypothesis on $S_{1}$ is also true for $S_{2}$. To get the desired conclusion, it is clear that we just have to prove that $\phi$ coincides on $X$ with some symmetry $f$ of $\mathcal{H}_{n}$.

- Then, let us remark that an elementary undirected cycle of length 4 on the edges of $\mathcal{H}_{n}$ is necessarily built on the 4 edges of a 2-dimensional face of $\mathcal{H}_{n}$. Thus $\phi$ associates to the 4 nodes of a 2-dimensional face of $\mathcal{H}_{n}$ the 4 nodes of a 2 -dimensional face of $\mathcal{H}_{n}$; the same for any symmetry $f$ of $\mathcal{H}_{n}$. This proves that if $\phi$ and $f$ coincide on 3 nodes of a 2 -dimensional face of $\mathcal{H}_{n}$, then they coincide on the fourth one, since a 2 -dimensional face is entirely determined by three of its nodes. 
- Let the $\overrightarrow{e_{i}}$ be the vectors of the canonical basis of $\mathbb{R}^{n}, A=(0, \ldots, 0)$, $A^{\prime}=\phi(A)$ and $A_{i}=A+\overrightarrow{e_{i}}$. By the hypothesis, there exists a permutation $\sigma$ of $\{1, \ldots, n\}$ and $\left(\varepsilon_{1}, \ldots, \varepsilon_{n}\right) \in\{-1,+1\}^{n}$ such that $\phi\left(A_{i}\right)=A^{\prime}+\varepsilon_{\sigma(i)} \overrightarrow{e_{\sigma(i)}}$, for $i=1, \ldots, n$. It is easily seen that there exists a unique symmetry $f$ of $\mathcal{H}_{n}$ such that $f(A)=A^{\prime}$ and $\vec{f}\left(\overrightarrow{e_{i}}\right)=\varepsilon_{\sigma(i)} \overrightarrow{e_{\sigma(i)}}$. Hence $\phi$ and $f$ coincide on $A$ and on the $n$ points $A_{i}$, which are the $n$ nodes of $\mathcal{H}_{n}$ with exactly one coordinate equal to 1 .

- For $k \geq 1$ and a subset $\left\{i_{1}, \ldots, i_{k}\right\}$ of $\{1, \ldots, n\}$ with $k$ elements, let us denote by $A_{i_{1}, \ldots, i_{k}}$ the point $A+\overrightarrow{i_{1}}+\cdots+\overrightarrow{i_{k}}$. Suppose that $\phi$ and $f$ coincide on the nodes of $\mathcal{H}_{n}$ with at most $k$ coordinates equal to 1 , and let us consider a point $A_{i_{1}, \ldots, i_{k+1}}$. Then $A_{i_{1}, \ldots, i_{k-1}}, A_{i_{1}, \ldots, i_{k-1}, i_{k}}, A_{i_{1}, \ldots, i_{k-1}, i_{k+1}}$ and $A_{i_{1}, \ldots, i_{k+1}}$ are the 4 nodes of a 2-dimensional face of $\mathcal{H}_{n}$. The initial remark proves that $\phi$ and $f$ coincide on $A_{i_{1}, \ldots, i_{k+1}}$, and a finite iteration thus completes the proves.

Remark 2. In terms of regulations, the hypothesis on $\mathcal{G}_{a}\left(S_{1}\right)$ means the absence of self-activation of the components.

\subsection{Effect of a symmetry of the hypercube on regulatory graphs and logical rules}

From a boolean FDS, one can compute the RG and the logical rules. Now, given two isometric boolean FDS, we want to understand how their relative RG and logical rules look like.

Let us consider a boolean FDS $S: X \rightarrow X$ and an element $f$ of the group $\Gamma\left(\mathcal{H}_{n}\right)$. We aim to describe the effect of the action of $f$ on $\mathcal{R G}(S)$, including local graphs, paths and circuits, and the effect on logical rules.

As mentioned above, $f$ is the composition of a permutation $f_{1}$ of the axis and a product $f_{2}$ of hyperplane symmetries. In other words, $f=f_{2} \circ f_{1}$ where

$$
\begin{aligned}
& f_{1}\left(x_{1}, \ldots, x_{n}\right)=\left(x_{\sigma^{-1}(1)}, \ldots, x_{\sigma^{-1}(n)}\right) \text { and } \overrightarrow{f_{1}}\left(\overrightarrow{e_{i}}\right)=\overrightarrow{e_{\sigma(i)}}, \\
& f_{2}\left(x_{1}, \ldots, x_{n}\right)=\left(x_{1}^{\varepsilon_{1}}, \ldots, x_{n}^{\varepsilon_{n}}\right) \text { and } \overrightarrow{f_{2}}\left(\overrightarrow{e_{i}}\right)=\varepsilon_{i} \overrightarrow{e_{i}},
\end{aligned}
$$

which leads to $f(x)=\left(x_{\sigma^{-1}(1)}^{\varepsilon_{1}}, \ldots, x_{\sigma^{-1}(n)}^{\varepsilon_{n}}\right)$ and $\vec{f}\left(\overrightarrow{e_{i}}\right)=\varepsilon_{\sigma(i)} \overrightarrow{e_{\sigma(i)}}$.

Let us remark that the effect of $f_{1}$ just makes a renumbering of the axis, that will provide in the following a renumbering of the components $g_{i}$. 


\subsubsection{Effect on local and global regulatory graphs}

Given a state $x$, we are going to see that each interaction of the local graph $\mathcal{R G}_{x}(S)$ will turn under the isometry into an interaction between the corresponding renumbered components. The sign of this interaction is changed according to the hyperplane symmetries on the components involved, in a way that we precise below.

Proposition 3. In the previous notation, let $y$ be a state and $x=f^{-1}(y)$. The interactions of the local graph $\mathcal{R G}_{y}\left(\phi_{f}(S)\right)$ are obtained from the interactions of the local graph $\mathcal{R G}_{x}(S)$ in the following way: each interaction from $g_{i}$ to $g_{j}$ of sign $\alpha$ in $\mathcal{R G}_{x}(S)$ provides an interaction from $g_{\sigma(i)}$ to $g_{\sigma(j)}$ of sign $\alpha \varepsilon_{\sigma(i)} \varepsilon_{\sigma(j)}$ in $\mathcal{R G}_{y}\left(\phi_{f}(S)\right)$.

Proof. There is an interaction from $g_{i}$ to $g_{j}$ of sign $\alpha$ in $\mathcal{R G}_{x}(S)$ for each couple $(i, j)$ such that

$$
S\left(\bar{x}^{i}\right)=S\left(x+(-1)^{x_{i}} \overrightarrow{e_{i}}\right)=S(x)+(-1)^{x_{i}} \alpha \overrightarrow{e_{j}}+\vec{u} \quad(*),
$$

where $\vec{u} \perp \overrightarrow{e_{j}}$.

Considering the fact that $f(x)=y$ gives $f\left(\bar{x}^{i}\right)=y+(-1)^{x_{i}} \varepsilon_{\sigma(i)} \overrightarrow{e_{\sigma(i)}}$, and applying $f$ to $\left(^{*}\right)$, we get

$$
\phi_{f}(S)\left(y+(-1)^{x_{i}} \varepsilon_{\sigma(i)} \overrightarrow{e_{\sigma(i)}}\right)=\phi_{f}(S)(y)+(-1)^{x_{i}} \alpha \varepsilon_{\sigma(j)} \overrightarrow{e_{\sigma(j)}}+\vec{v},
$$

where $\vec{v} \perp \overrightarrow{e_{\sigma(j)}}$.

Thus there is an interaction from $g_{\sigma(i)}$ to $g_{\sigma(j)}$ of $\operatorname{sign} \alpha \varepsilon_{\sigma(i)} \varepsilon_{\sigma(j)}$ in $\mathcal{R G}_{y}\left(\phi_{f}(S)\right)$, and conversely considering $f^{-1}$.

This gives the important following corollary.

Proposition 4. The regulatory graphs $\mathcal{R G}(S)$ and $\mathcal{R G}\left(\phi_{f}(S)\right)$ are isomorphic directed graphs.

\subsubsection{Effect on paths and circuits}

The following proposition states that any path in the RG of $S$ is transported in the RG under the action of $f$. 
Proposition 5. Let $g_{i_{1}}, \ldots, g_{i_{r}}$ be a path of $\mathcal{R G}(S)$, and for each $k \in$ $\{1, \ldots, r-1\}$ a pair of states $\left\{x^{(k)},{\overline{x^{(k)}}}^{i_{k}}\right\}$ where the interaction from $g_{i_{k}}$ to $g_{i_{k+1}}$ is expressed with sign $\alpha_{k}$. Under the action of $f$, this path provides a path $g_{\sigma\left(i_{1}\right)}, \ldots, g_{\sigma\left(i_{r}\right)}$ in $\mathcal{R G}\left(\phi_{f}(S)\right)$, and for each $k \in\{1, \ldots, r-1\}$ a pair of states $\left\{y^{(k)}, \bar{y}^{(k)}{ }^{\sigma\left(i_{k}\right)}\right\}$ where the interaction from $g_{\sigma\left(i_{k}\right)}$ to $g_{\sigma\left(i_{k+1}\right)}$ is expressed with sign $\alpha_{k} \varepsilon_{\sigma\left(i_{k}\right)} \varepsilon_{\sigma\left(i_{k+1}\right)}$.

Proof. This proposition is an immediate consequence of Proposition 3, considering the local graphs $\mathcal{R} \mathcal{G}_{x_{k}}(S)$ and $y^{(k)}=f\left(x^{(k)}\right)$.

The following corollary states that the action of a hypercube symmetry does not change the signs of the circuits (see also [21], with more symbolic arguments).

Corollary 1. Let $g_{i_{1}}, \ldots, g_{i_{r}}$ be a circuit of $\mathcal{R G}(S)$, and for each $k \in$ $\{1, \ldots, r\}$ a pair of states $\left\{x^{(k)}, \bar{x}^{(k)}{ }^{i}{ }_{k}\right\}$ where the interaction from $g_{i_{k}}$ to $g_{i_{k+1}}$ is expressed with sign $\alpha_{k}$ (with the notation $i_{r+1}=i_{1}$ ). Let $\alpha$ denote $\prod_{k=1}^{r} \alpha_{k}$. Under the action of $f$, the circuit $g_{i_{1}}, \ldots, g_{i_{r}}$ provides a circuit $g_{\sigma\left(i_{1}\right)}, \ldots, g_{\sigma\left(i_{r}\right)}$ in $\mathcal{R G}\left(\phi_{f}(S)\right)$, and for each $k \in\{1, \ldots, r\}$ a pair of states $\left\{y^{(k)}, \bar{y}^{(k)}{ }^{\sigma\left(i_{k}\right)}\right\}$ where the interaction from $g_{\sigma\left(i_{k}\right)}$ to $g_{\sigma\left(i_{k+1}\right)}$ is expressed with sign $\beta_{k}$ such that $\prod_{k=1}^{r} \beta_{k}=\alpha$.

Proof. From Proposition 5 we get $\beta_{k}=\alpha_{k} \varepsilon_{\sigma\left(i_{k}\right)} \varepsilon_{\sigma\left(i_{k+1}\right)}$, and thus $\prod_{k=1}^{r} \beta_{k}=$ $\prod_{k=1}^{r} \alpha_{k}\left(\prod_{k=1}^{r} \varepsilon_{\sigma\left(i_{k}\right)}\right)^{2}=\alpha$.

\subsubsection{Effect on logical rules}

We are going to consider a component $g_{i_{0}}$, and $C\left(g_{i_{0}}\right)$ a logical rule for $g_{i_{0}}$ with respect to $S$. Let us recall that in this logical rule the components are allowed to be in the restricted subset of the regulators of $g_{i_{0}}$, which we assume in the sequel. We deal with how to compute a logical rule $C_{f}\left(g_{\sigma\left(i_{0}\right)}\right)$ for $g_{\sigma\left(i_{0}\right)}$ with respect to $\phi_{f}(S)$.

With the convention $\neg \neg=\nu$, where $\nu$ is the empty word, we have the following proposition.

Proposition 6. Let $g_{i_{0}}$ be a component, and $C\left(g_{i_{0}}\right)$ a logical rule for $g_{i_{0}}$ with respect to $S$ in the following normal disjunctive form:

$$
C\left(g_{i_{0}}\right)=\bigvee_{j \in\{1, \ldots, s\}} \bigwedge_{k \in\left\{1, \ldots, r_{j}\right\}} w_{j, k} g_{i_{j, k}},
$$


where the $g_{i_{j, k}}$ are regulators of $g_{i_{0}}$ and the $w_{j, k}$ are equal to the empty word $\nu$ or to $\neg$.

1. If $\varepsilon_{\sigma\left(i_{0}\right)}=+1$,

$$
C_{f}\left(g_{\sigma\left(i_{0}\right)}\right)=\bigvee_{j \in\{1, \ldots, s\}} \bigwedge_{k \in\left\{1, \ldots r_{j}\right\}} w_{j, k} \lambda_{j, k} g_{\sigma\left(i_{j, k}\right)}
$$

where $\lambda_{j, k}=\left\{\begin{array}{ll}\nu & \text {, if } \varepsilon_{\sigma\left(i_{j, k}\right)}=+1 \\ \neg & \text {, if } \varepsilon_{\sigma\left(i_{j, k}\right)}=-1\end{array}\right.$, is a logical rule for $g_{\sigma\left(i_{0}\right)}$ with respect to $\phi_{f}(S)$.

2. If $\varepsilon_{\sigma\left(i_{0}\right)}=-1$,

$$
C_{f}\left(g_{\sigma\left(i_{0}\right)}\right)=\bigwedge_{j \in\{1, \ldots, s\}} \bigvee_{k \in\left\{1, \ldots r_{j}\right\}} w_{j, k} \mu_{j, k} g_{\sigma\left(i_{j, k}\right)}
$$

where $\mu_{j, k}=\left\{\begin{array}{ll}\neg & \text {, if } \varepsilon_{\sigma\left(i_{j, k}\right)}=+1 \\ \nu & \text {, if } \varepsilon_{\sigma\left(i_{j, k}\right)}=-1\end{array}\right.$, is a logical rule for $g_{\sigma\left(i_{0}\right)}$ with respect to $\phi_{f}(S)$ in a conjunctive normal form.

Proof. Let the logical rule $C\left(g_{i_{0}}\right)$ be expressed as

$$
C\left(g_{i_{0}}\right)=\bigvee_{j \in\{1, \ldots, s\}} \bigwedge_{k \in\left\{1, \ldots, r_{j}\right\}} w_{j, k} g_{i_{j, k}}
$$

In terms of subsets of $X$, this means that we have the following equality:

$$
\left\{x \in X ; S(x)_{i_{0}}=1\right\}=\bigcup_{j \leq s}\left\{x \in X ;\left(x_{i_{j, 1}}, \ldots, x_{i_{j, r_{j}}}\right)=\left(a_{j, 1}, \ldots, a_{j, r_{j}}\right)\right\}
$$

where $a_{j, k}=1$ if $w_{j, k}=\nu$ and $a_{j, k}=0$ if $w_{j, k}=\neg$.

Applying $f$ to both members of these equalities, and considering the fact that $f\left(x_{1}, \ldots, x_{n}\right)=\left(x_{\sigma(1)}^{\varepsilon_{\sigma(1)}}, \ldots, x_{\sigma(n)}^{\varepsilon_{\sigma(n)}}\right)$, we obtain:

$$
\begin{aligned}
& \left\{y \in X ; \phi_{f}(S)(y)_{\sigma\left(i_{0}\right)}=1^{\varepsilon_{\sigma\left(i_{0}\right)}}\right\} \\
& =\bigcup_{j \leq s}\left\{y \in X ;\left(y_{\sigma\left(i_{j, 1}\right)}, \ldots, y_{\sigma\left(i_{j, r_{j}}\right)}\right)=\left(a_{j, 1}^{\varepsilon_{\sigma\left(i_{j, 1}\right)}}, \ldots, a_{j, r_{j}}^{\varepsilon_{\sigma\left(i_{j, r_{j}}\right)}}\right)\right\}(*) .
\end{aligned}
$$


1. Suppose that $\varepsilon_{\sigma\left(i_{0}\right)}=+1$.

Then the equality $(*)$ can be reconverted right away in a logical rule $C_{f}\left(g_{\sigma\left(i_{0}\right)}\right)$ for $g_{\sigma\left(i_{0}\right)}$ :

$$
C_{f}\left(g_{\sigma\left(i_{0}\right)}\right)=\bigvee_{j \in\{1, \ldots, s\}} \bigwedge_{k \in\left\{1, \ldots r_{j}\right\}} W_{j, k} g_{\sigma\left(i_{j, k}\right)}
$$

where $W_{j, k}=\nu$ if $a_{j, k}^{\left.\varepsilon_{\sigma(i, k}\right)}=1$ and $W_{j, k}=\neg$ if $a_{j, k}^{\varepsilon_{\sigma\left(i_{j, k}\right)}}=0$.

In other words,

$W_{j, k}=w_{j, k} \lambda_{j, k}$, where $\lambda_{j, k}=\left\{\begin{array}{ll}\nu & , \text { if } \varepsilon_{\sigma\left(i_{j, k}\right)}=+1 \\ \neg & , \text { if } \varepsilon_{\sigma\left(i_{j, k}\right)}=-1\end{array}\right.$.

2. Suppose that $\varepsilon_{\sigma\left(i_{0}\right)}=-1$.

Like in the first case, the equality $(*)$ makes it possible to obtain a logical rule $C_{f}\left(g_{\sigma\left(i_{0}\right)}\right)$ for $g_{\sigma\left(i_{0}\right)}$ :

$$
C_{f}\left(g_{\sigma\left(i_{0}\right)}\right)=\neg\left[\bigvee_{j \in\{1, \ldots, s\}} \bigwedge_{k \in\left\{1, \ldots r_{j}\right\}} w_{j, k} \lambda_{j, k} g_{\sigma\left(i_{j, k}\right)}\right],
$$

where $\lambda_{j, k}=\left\{\begin{array}{ll}\nu & , \text { if } \varepsilon_{\sigma\left(i_{j, k}\right)}=+1 \\ \neg & \text {, if } \varepsilon_{\sigma\left(i_{j, k}\right)}=-1\end{array}\right.$.

Hence, it turns out that we obtain $C_{f}\left(g_{\sigma\left(i_{0}\right)}\right)$ as the conjunction of disjunctive clauses

$$
C_{f}\left(g_{\sigma\left(i_{0}\right)}\right)=\bigwedge_{j \in\{1, \ldots, s\}} \bigvee_{k \in\left\{1, \ldots r_{j}\right\}} \neg w_{j, k} \lambda_{j, k} g_{\sigma\left(i_{j, k}\right)}
$$

that is

$$
C_{f}\left(g_{\sigma\left(i_{0}\right)}\right)=\bigwedge_{j \in\{1, \ldots, s\}} \bigvee_{k \in\left\{1, \ldots r_{j}\right\}} w_{j, k} \mu_{j, k} g_{\sigma\left(i_{j, k}\right)},
$$

where $\mu_{j, k}=\left\{\begin{array}{ll}\neg & , \text { if } \varepsilon_{\sigma\left(i_{j, k}\right)}=+1 \\ \nu & , \text { if } \varepsilon_{\sigma\left(i_{j, k}\right)}=-1\end{array}\right.$. 
Remark 3. Let us suppose that a logical formula for the negation of $C\left(g_{i_{0}}\right)$ is available in a disjunctive normal form

$$
\neg C\left(g_{i_{0}}\right)=\bigvee_{j \in\left\{1, \ldots, s^{\prime}\right\}} \bigwedge_{k \in\left\{1, \ldots, r_{j}^{\prime}\right\}} w_{j, k}^{\prime} g_{i_{j, k}^{\prime}},
$$

where the $g_{i_{j, k}^{\prime}}$ are regulators of $g_{i_{0}}$ and the $w_{j, k}^{\prime}$ are equal to the empty word $\nu$ or to $\neg$. In a similar way to the above proof, it can be shown that if $\varepsilon_{\sigma\left(i_{0}\right)}=-1$,

$$
C_{f}\left(g_{\sigma\left(i_{0}\right)}\right)=\bigvee_{j \in\left\{1, \ldots, s^{\prime}\right\}} \bigwedge_{k \in\left\{1, \ldots r_{j}^{\prime}\right\}} w_{j, k}^{\prime} \mu_{j, k}^{\prime} g_{\sigma\left(i_{j, k}\right)},
$$

where $\mu_{j, k}^{\prime}=\left\{\begin{array}{ll}\nu & \text { if } \varepsilon_{\sigma\left(i_{j, k}^{\prime}\right)}=+1 \\ \neg & \text { if } \varepsilon_{\sigma\left(i_{j, k}^{\prime}\right)}=-1\end{array}\right.$,

is a logical rule for $g_{\sigma\left(i_{0}\right)}$ with respect to $\phi_{f}(S)$ in a disjunctive normal form.

Example 1. 1. Let us place in the case where $f$ is the hyperplane symmetry transforming $\left(x_{1}, \ldots, x_{n}\right)$ into $\left(x_{1}, \ldots, x_{j_{0}-1}, \overline{x_{j_{0}}}, x_{j_{0}+1}, \ldots, x_{n}\right)$. That is $\sigma(i)=i$ for all $i \in\{1, \ldots, n\}, \varepsilon_{j_{0}}=-1$ and $\varepsilon_{i}=+1$ if $i \neq j_{0}$.

Then, for a component $g_{i_{0}}$ with logical formula

$$
C\left(g_{i_{0}}\right)=\bigvee_{j \in\{1, \ldots, s\}} \bigwedge_{k \in\left\{1, \ldots, r_{j}\right\}} w_{j, k} g_{i_{j, k}},
$$

if $i_{0} \neq j_{0}$, the logical formula $C_{f}\left(g_{i_{0}}\right)$ is obtained from $C\left(g_{i_{0}}\right)$ switching $w_{j, k}$ into $\neg w_{j, k}$ when $i_{j, k}=j_{0}$. In particular $C\left(g_{i_{0}}\right)$ remains unchanged if $g_{j_{0}}$ is not a regulator of $g_{i_{0}}$.

Moreover, if $i_{0}=j_{0}$, the logical formula $C_{f}\left(g_{j_{0}}\right)$ is obtained from $C\left(g_{j_{0}}\right)$ switching $w_{j, k}$ into $\neg w_{j, k}$ when $i_{j, k} \neq j_{0}$ and exchanging $\bigwedge$ and $\bigvee$. In particular, $C_{f}\left(g_{j_{0}}\right)$ is the negation of $C\left(g_{j_{0}}\right)$ if $g_{j_{0}}$ is not self-regulated.

2. Let us now consider the case where $f$ is the symmetry with respect to the center of $\mathcal{H}_{n}$. That means that for all $i \in\{1, \ldots, n\}$ we have $\vec{f}\left(\overrightarrow{e_{i}}\right)=-\overrightarrow{e_{i}}$, or equivalently $\sigma(i)=i$ and $\varepsilon_{i}=-1$.

Then, for each component $g_{i_{0}}$, given a logical formula

$$
C\left(g_{i_{0}}\right)=\bigvee_{j \in\{1, \ldots, s\}} \bigwedge_{k \in\left\{1, \ldots, r_{j}\right\}} w_{j, k} g_{i_{j, k}},
$$


we have

$$
C_{f}\left(g_{i_{0}}\right)=\bigwedge_{j \in\{1, \ldots, s\}} \bigvee_{k \in\left\{1, \ldots, r_{j}\right\}} w_{j, k} g_{i_{j, k}} .
$$

For example, the action of $f$ exchanges chorded circuits "AND" and "OR" from one to the other (see 4.2 for more details).

\subsection{A useful working tool}

We aim at classify the set of all the boolean FDS on the basis of the symmetries of the hypercube, defining classes gathering isometric FDS. We have emphasized the common features of the conjugates $\phi_{f}(S)$ of a given boolean FDS $S$ under these symmetries, through regulatory graphs and logical rules ; we can then restrict the dynamical analysis of all the boolean FDS to one representative per class. The following property is a key ingredient to guide the choice of an appropriate representative.

In the proof, we will consider directed graphs $T=(V, E)$ such that the undirected graph $\tilde{T}=(V,\{\{a, b\} ;(a, b) \in E$ and $a \neq b\})$ is a tree, that is an undirected acyclic connected graph. We will say that $T$ is tree-supported if $\tilde{T}$ is a tree and if $T$ does not include both an edge $(a, b)$ and its reverse $(b, a)$ (in particular nor any loop $(a, a)$ ), and we will call leaves of $T$ the leaves of $\tilde{T}$.

Proposition 7. Let $S$ a boolean $F D S$, and $\mathcal{R G}(S)$ its corresponding regulatory graph. Consider a subgraph $T=(V, E)$ of $\mathcal{R G}(S)$ which is treesupported. Given any family of signs $\left(\alpha_{e}\right)_{e \in E}$ and $g_{i} \in V$, there exists a symmetry $f$ of the hypercube such that every interaction e in $E$ can be expressed in $\mathcal{R} \mathcal{G}\left(\phi_{f}(S)\right)$ with sign $\alpha_{e}$ and such that $i \notin\left\{k \in\{1, \ldots, n\} ; \vec{f}\left(\overrightarrow{e_{k}}\right)=-\overrightarrow{e_{k}}\right\}$.

Proof. We use an induction argument on $\# V$. If $T$ has zero or one vertex, $E$ is empty and we can take $f$ as the identity function. We suppose that the result holds for any tree-supported subgraph $T$ of $\mathcal{R G}(S)$ with $m$ vertices, $m>0$. If $T$ has $m+1$ vertices, it has at least two vertices and thus at least two leaves. Let $g_{j}$ be one of them which is not $g_{i}$; the vertex $g_{j}$ is involved in exactly one edge $e^{\prime}$ of $T$. Let $\left(\alpha_{e}\right)_{e \in E}$ be a family of signs. The treesupported subgraph $T^{\prime}=\left(V \backslash\left\{g_{j}\right\}, E \backslash\left\{e^{\prime}\right\}\right)$ of $\mathcal{R} \mathcal{G}(S)$ has $m$ vertices, thus there exists $f \in \Gamma\left(\mathcal{H}_{n}\right)$, product of hyperplane symmetries, such that every interaction $e$ in $E \backslash\left\{e^{\prime}\right\}$ can be expressed in $\mathcal{R} \mathcal{G}\left(\phi_{f}(S)\right)$ with the sign $\alpha_{e}$, and $i, j \notin\left\{k \in\{1, \ldots, n\} ; \vec{f}\left(\overrightarrow{e_{k}}\right)=-\overrightarrow{e_{k}}\right\}$. If the interaction $e^{\prime}$ can be expressed in $\mathcal{R G}\left(\phi_{f}(S)\right)$ with the sign $\alpha_{e^{\prime}}$, the symmetry $f$ fulfills our expectations. If not, it means that $e^{\prime}$ is expressed in $\mathcal{R} \mathcal{G}\left(\phi_{f}(S)\right)$ with the sign $-\alpha_{e^{\prime}}$. In this 


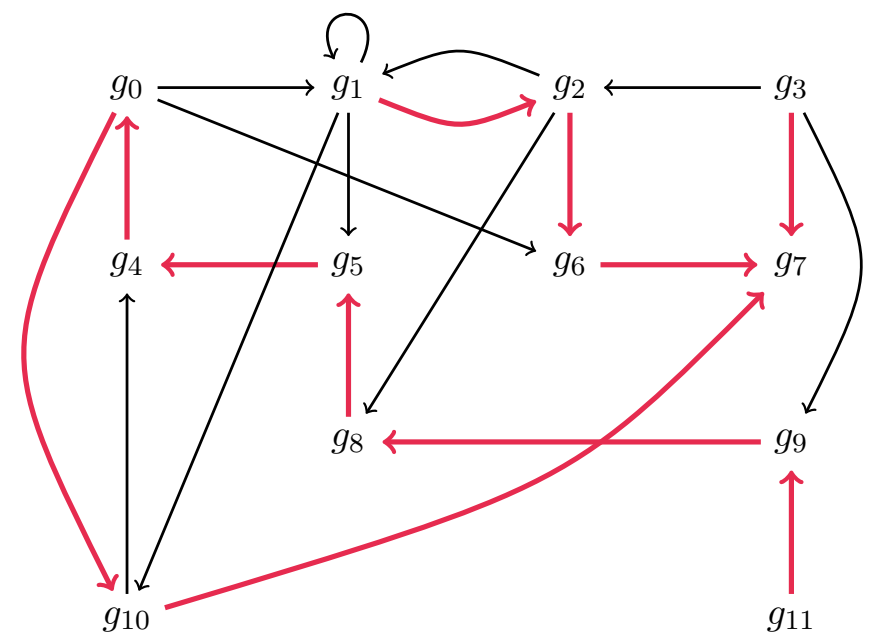

Figure 2: Proposition 7 can be applied on the colored subgraph.

case, the (commutative) composition $f^{\prime}$ of $f$ and the hyperplane symmetry $x \mapsto \bar{x}^{j}$ works, according to Proposition 3 .

\section{Application to regulatory motifs analysis}

As mentioned in the introduction, boolean FDS are used for the modeling of gene regulatory networks. The analysis of the model is difficult, mainly because of combinatorial explosions to generate the STG. Motifs analysis is a way to capture local properties of the dynamics without generating the STG. Indeed, it is well-known that some specific motifs, i.e. specific topological components embedded in the $\mathrm{RG}$, play a major role allowing the presence and/or the maintain of complex dynamical properties (as multistationnarity, or sustained oscillations). Several studies of well-known motifs have been undertaken in the context of boolean logical modeling: isolated circuits [12], chorded circuits [11], flower-graphs, and more generally hub-graphs [3].

As an illustration, it is possible to study boolean dynamics of well-known motifs - isolated circuits, chorded circuits, flower-graphs, and more generally hub-graphs - through the choice of favorite representatives.

Let us recall that for $a \in\{0,1\}$, the notation $a^{\alpha}$ means $a$ when $\alpha=1$ 

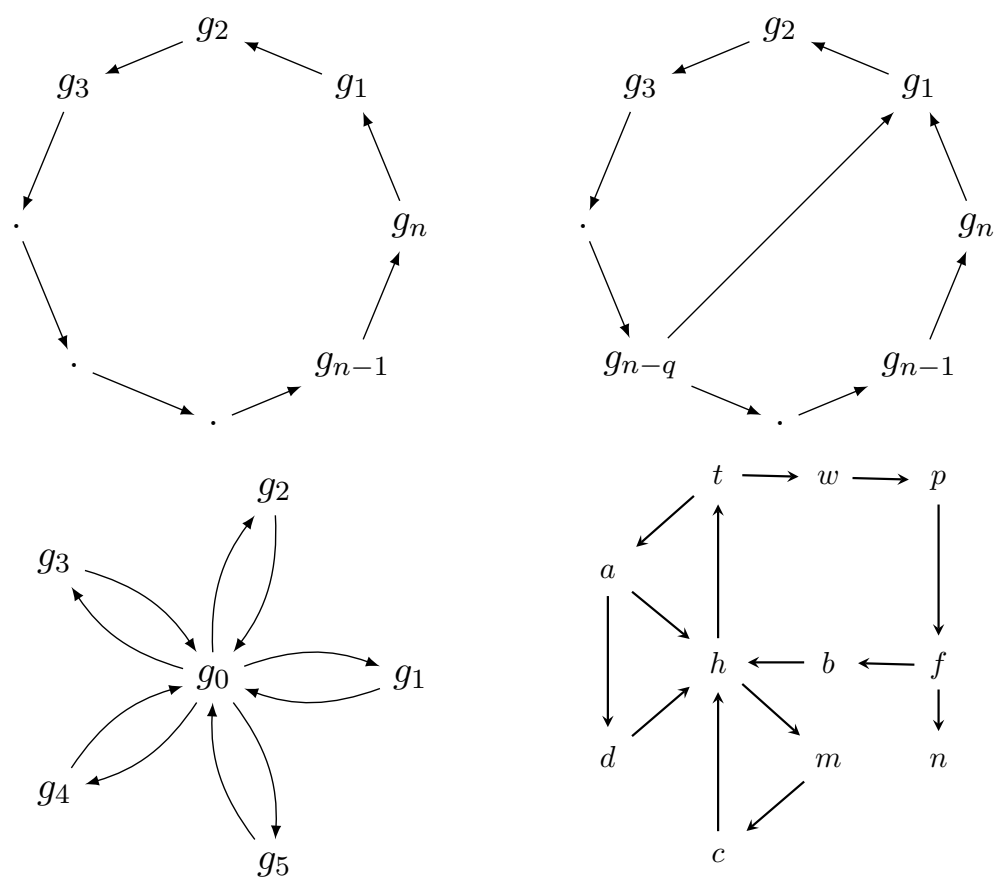

Figure 3: Isolated circuit (up, left), chorded circuit (up, right), flower-graph (down, left), hub-graph (down, right).

and $1-a$ when $\alpha=-1$.

\subsection{Isolated circuits}

We consider boolean FDS whose associated RG are isolated circuits on $n$ components $g_{1}, \ldots, g_{n}$, with $n \geq 2$ (see Figure 3 ).

In this particular case, the signs of the interactions do not depend on the states where they are expressed (cf. Proposition 1). If $\alpha_{k}$ denotes the sign of the interaction from $g_{k}$ to $g_{k+1}$, for $k=1, \ldots, n$ (where $g_{n+1}=g_{1}$ ), we will denote by $\mathcal{C}_{n}\left(\alpha_{1}, \ldots, \alpha_{n}\right)$ the isolated circuit associated to the FDS

$$
\left(x_{1}, \ldots, x_{n}\right) \mapsto\left(x_{n}^{\alpha_{n}}, x_{1}^{\alpha_{1}}, \ldots, x_{n-1}^{\alpha_{n-1}}\right) .
$$

In other words, $C\left(g_{k+1}\right)=w_{k} g_{k}$, where $w_{k}=\nu$ if $\alpha_{k}=+1$, and $w_{k}=\neg$ if $\alpha_{k}=-1$, is a logical rule for the component $g_{k+1}$. 
Proposition 8. Given an integer $n \geq 1$, there exists exactly two classes of isometric boolean FDS whose $R G$ are respectively positive and negative isolated circuits of length $n$.

Proof. First of all, the symmetries of $\Gamma\left(\mathcal{H}_{n}\right)$ transform isolated circuits of length $n$ into isolated circuits of length $n$ (cf. Proposition 4). Let us consider the path $T$ from $g_{1}$ towards $g_{n}$ obtained from $\mathcal{C}_{n}\left(\alpha_{1}, \ldots, \alpha_{n}\right)$ by removing the interaction of $g_{n}$ on $g_{1}$ (see Figure 4). Following Proposition 7, $T$ is tree-supported, and there exists a symmetry $f \in \Gamma\left(\mathcal{H}_{n}\right)$ which transforms $\mathcal{C}_{n}\left(\alpha_{1}, \ldots, \alpha_{n}\right)$ into $\mathcal{C}_{n}\left(+1, \ldots,+1, \prod_{k=1}^{n} \alpha_{k}\right)$, the action of $f$ preserving signs of circuits (cf. Corollary 1). Hence we have two favorite representatives for the isolated circuits of length $n$, the boolean dynamics of $\mathcal{C}_{n}(+1, \ldots,+1)$ and the one of $\mathcal{C}_{n}(+1, \ldots,+1,-1)$, which are not isometric, due to the invariance of the sign in a class; thus they represent exactly two classes.

Let us recall that the attractors of the synchronous and the asynchronous dynamics of isolated circuits are well-known; in particular the asynchronous dynamics involves two stable states in the case of positive circuits, and one cyclic attractor in the case of negative circuits [11].

\subsection{Chorded circuits}

Remark 4. In this paragraph, the $R G$ will involve a component with exactly two regulators. This brings us to precise in a preliminary examination the boolean functions $B:\{0,1\}^{2} \rightarrow\{0,1\}$ whose truth tables are the ones of the logical rules of components with two regulators. Among the 16 functions $B:\{0,1\}^{2} \rightarrow\{0,1\},(a, b) \mapsto B(a, b)$, two are constant and four depend on a single argument $a$ or $b$. Hence there are 10 possible values for $B$, given by:

- $a \wedge b, a \wedge \neg b, \neg a \wedge b, \neg a \wedge \neg b$,

- $a \vee b, a \vee \neg b, \neg a \vee b, \neg a \vee \neg b$,

- $a \oplus b=\neg a \oplus \neg b, a \oplus \neg b=\neg a \oplus b$

(where $a \oplus b=(a \wedge \neg b) \vee(\neg a \wedge b))$.

In the following, the presence or absence of negations will be carried by parameters $\alpha_{k}$ and $\alpha_{k}^{(s)}$, and therefore we will fix $B$ in $\{\wedge, \vee, \oplus\}$.

We consider now boolean FDS whose RG are chorded circuits on $n$ components $g_{1}, \ldots, g_{n}$, the component $g_{1}$ being the target of a shortcut (see Figure 3). 
More precisely, let us denote by $\mathcal{C C}_{n, q}^{B}\left(\alpha_{1}, \ldots, \alpha_{n} ; \alpha_{n-q}^{(s)}\right)$ the chorded circuit associated to the FDS

$$
\left(x_{1}, \ldots, x_{n-q}, \ldots, x_{n}\right) \mapsto\left(B\left(x_{n}^{\alpha_{n}}, x_{n-q}^{\alpha_{n-q}^{(s)}}\right), x_{1}^{\alpha_{1}}, \ldots, x_{n-1}^{\alpha_{n-1}}\right),
$$

with $q \geq 1$ and $n \geq q+1, \alpha_{i} \in\{ \pm 1\}$ for $i \in\{1, \ldots n\}, \alpha_{n-q}^{(s)} \in\{ \pm 1\}$, and $B \in\{$ AND, OR, XOR $\}=\{\wedge, \vee, \oplus\}$ (following Remark 4 ).

In terms of logical rules:

- $C\left(g_{k+1}\right)=w_{k} g_{k}$, where $w_{k}=\nu$ if $\alpha_{k}=+1$, and $w_{k}=\neg$ if $\alpha_{k}=-1$, is a logical rule for the component $g_{k+1}$, for $k \in\{1, \ldots, n-1\}$,

- $C\left(g_{1}\right)=B\left(w_{n} g_{n}, w_{n-q}^{(s)} g_{n-q}\right)$, where $w_{n}=\nu$ if $\alpha_{n}=+1, w_{n}=\neg$ if $\alpha_{n}=-1, w_{n-q}^{(s)}=\nu$ if $\alpha_{n-q}^{(s)}=+1$, and $w_{n-q}=\neg$ if $\alpha_{n-q}^{(s)}=-1$, is a logical rule for the component $g_{1}$.

For $k \neq 1$, the sign of the interaction towards $g_{k}$ does not depend on the states where it is expressed, due to the fact that $g_{k}$ has only one regulator, and this sign is equal to $\alpha_{k-1}$ (cf. Proposition 1). Moreover, it is easy to check that if $B \in\{\mathrm{AND}, \mathrm{OR}\}$ the signs of the interaction from $g_{n}$ to $g_{1}$ and from $g_{n-q}$ to $g_{1}$ are respectively equal to $\alpha_{n}$ and $\alpha_{n-q}^{(s)}$, and that, at the contrary, these signs depend on the local graphs if $B=$ xOR.

The chorded circuit $\mathcal{C C}_{n, q}^{B}\left(\alpha_{1}, \ldots, \alpha_{n} ; \alpha_{n-q}^{(s)}\right)$ is built of two embedded circuits, $\mathcal{C}_{n}\left(\alpha_{1}, \ldots, \alpha_{n}\right)$ and $\mathcal{C}_{n-q}\left(\alpha_{1}, \ldots, \alpha_{n-q-1}, \alpha_{n-q}^{(s)}\right)$, in the notation of 4.1. Let $\varsigma=\prod_{k=1}^{n} \alpha_{k}$ and $\varsigma^{(s)}=\prod_{k<n-q} \alpha_{k} \alpha_{n-q}^{(s)}$ be the signs of these two isolated circuits; they play a significant role in the case $B \in\{$ AND, OR $\}$.

Proposition 9. Given integers $q \geq 1$ and $n \geq q+1$, there exists exactly five classes of isometric boolean FDS whose $R G$ are the chorded circuits $\mathcal{C C}_{n, q}^{B}\left(\alpha_{1}, \ldots, \alpha_{n} ; \alpha_{n-q}^{(s)}\right)$, with $B \in\{\mathrm{AND}, \mathrm{OR}, \mathrm{XOR}\}$ :

- four classes gather the boolean FDS related to the chorded circuits $\mathcal{C C}_{n, q}^{B}\left(\alpha_{1}, \ldots, \alpha_{n} ; \alpha_{n-q}^{(s)}\right)$ with $B \in\{\mathrm{AND}, \mathrm{OR}\}$, according to the value of $\left(\varsigma, \varsigma^{(s)}\right)$ in $\{ \pm 1\}^{2}$;

- one class gathers the boolean FDS related to the chorded circuits $\mathcal{C C}_{n, q}^{\mathrm{XOR}}\left(\alpha_{1}, \ldots, \alpha_{n} ; \alpha_{n-q}^{(s)}\right)$.

Proof. The Proposition 4 shows that the symmetries of $\Gamma\left(\mathcal{H}_{n}\right)$ transform chorded circuits into chorded circuits with same values of $n$ and $q$.

Let us consider $\mathcal{C C}_{n, q}^{B}\left(\alpha_{1}, \ldots, \alpha_{n} ; \alpha_{n-q}^{(s)}\right)$. The path $T$ from $g_{1}$ towards $g_{n}$ obtained by removing the two interactions on $g_{1}$ is tree-supported (see 
Figure 4). Following Proposition 7 and Remark 4, there exists a symmetry $f \in \Gamma\left(\mathcal{H}_{n}\right)$ changing $\mathcal{C C}_{n, q}^{B}\left(\alpha_{1}, \ldots, \alpha_{n} ; \alpha_{n-q}^{(s)}\right)$ into $\mathcal{C C}_{n, q}^{B^{\prime}}(+1, \ldots,+1, \pm 1 ; \pm 1)$, with $B^{\prime} \in\{$ AND, OR, XOR $\}$.

- If $B \in\{\mathrm{AND}, \mathrm{OR}\}$, the fact that the signs of the two embedded circuits are determined and the Proposition 3 show that $B^{\prime} \in\{$ AND, OR $\}$. Given this and the invariance of $\left(\varsigma, \varsigma^{(s)}\right)$ under the action of $f$ (cf. Corollary 1), the symmetry $f$ transforms $\mathcal{C C}_{n, q}^{B}\left(\alpha_{1}, \ldots, \alpha_{n} ; \alpha_{n-q}^{(s)}\right)$ into $\mathcal{C C}_{n, q}^{B^{\prime}}\left(+1, \ldots,+1, \varsigma ; \varsigma^{(s)}\right)$. Moreover, the Remark 3 shows that $\mathcal{C C}_{n, q}^{\mathrm{AND}}\left(+1, \ldots,+1, \varsigma ; \varsigma^{(s)}\right)$ and $\mathcal{C C}_{n, q}^{\mathrm{OR}}\left(+1, \ldots,+1, \varsigma ; \varsigma^{(s)}\right)$ are isometric. Hence, for example, $\mathcal{C C}_{n, q}^{\text {AND }}\left(+1, \ldots,+1, \varsigma ; \varsigma^{(s)}\right)$ gives, according to the four possible values of $\left(\varsigma, \varsigma^{(s)}\right)$, four favorite representatives "AND". They are not isometric because of the invariance of $\left(\varsigma, \varsigma^{(s)}\right)$ in a class, and thus represent exactly four classes.

- If $B=$ XOR, the previous study shows that the resultant classes will be disjoined from the case $B \in\{\mathrm{AND}, \mathrm{OR}\}$, and therefore $B^{\prime}=B$. Moreover the equalities $a \oplus b=\neg a \oplus \neg b$ and $a \oplus \neg b=\neg a \oplus b$ show that $\mathcal{C C}_{n, q}^{\mathrm{XOR}}(+1, \ldots,+1,+1 ;+1)$ and $\mathcal{C C}_{n, q}^{\mathrm{XOR}}(+1, \ldots,+1,-1 ;-1)$ are related to the same FDS, and it is the same for $\mathcal{C C}_{n, q}^{\mathrm{XOR}}(+1, \ldots,+1,-1 ;+1)$ and $\mathcal{C C}_{n, q}^{\mathrm{xOR}}(+1, \ldots,+1,+1 ;-1)$. Finally, the Remark 3 shows that $\mathcal{C C}_{n, q}^{\mathrm{XOR}}(+1, \ldots,+1,+1 ;+1)$ and $\mathcal{C C}_{n, q}^{\mathrm{XOR}}(+1, \ldots,+1,+1 ;-1)$ are isometric, since $a \oplus b=(a \wedge \neg b) \vee(\neg a \wedge b)$, and $a \oplus \neg b=(a \vee \neg b) \wedge(\neg a \vee b)$. Thus $\mathcal{C C}_{n, q}^{\mathrm{XOR}}(+1, \ldots,+1,+1 ;+1)$ represent the single class.

A study of the synchronous and the asynchronous dynamics of chorded circuits can be found in $[13,11]$.

If $B \in\{$ AND, OR $\}$, a chorded circuit is said coherent if $\varsigma=\varsigma^{(s)}$, and incoherent otherwise. It is shown for example that in the asynchronous dynamics a coherent chorded circuit and its corresponding long circuit have the same number and type of attractors, and that an incoherent chorded circuit has a unique attractor, which is a stable state. If $B=\mathrm{xOR}$, the asynchronous dynamics involves a unique attractor, which is a stable state.

\subsection{Flower-graphs}

In this section, we study boolean FDS whose RG are specific motives, called flower-graphs. A flower-graph with $n$ petals is a directed graph $(V, E)$ such that $V$ has $n+1$ elements, and there exists a particular node $g_{0} \in V$ called 

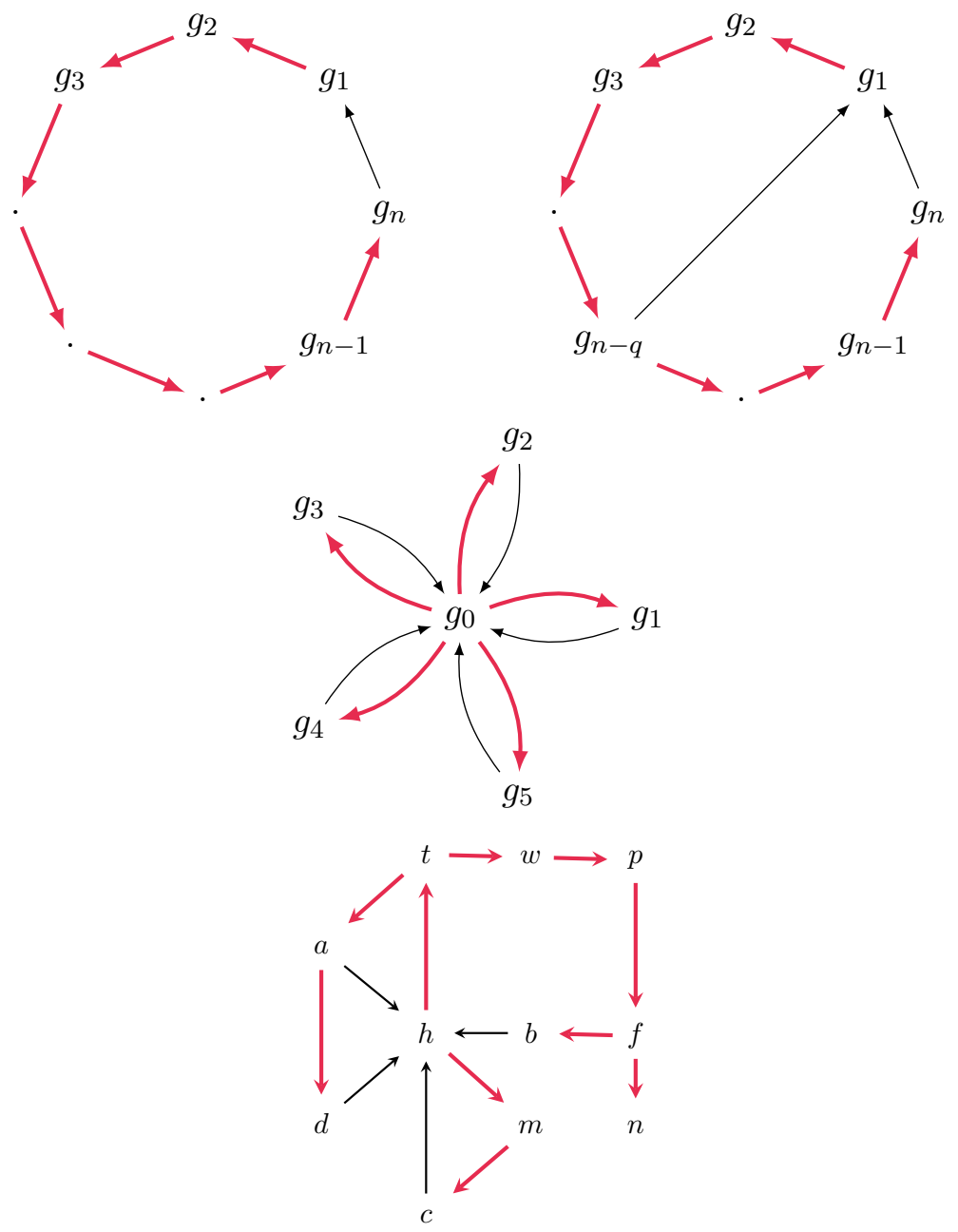

Figure 4: Proposition 7 is applied on the colored subgraphs. 
hub for which $E=\left\{\left(g_{0}, g\right) \cup\left(g, g_{0}\right) ; g \in V \backslash\left\{g_{0}\right\}\right\}$ (see Figure 3). We suppose moreover that $n \geq 2$ (otherwise $(V, E)$ is a circuit).

Let $S$ be a boolean FDS whose associated regulatory graph $\mathcal{R G}(S)$ is a flower-graph, with hub $g_{0}$ and $V=\left\{g_{0}, \ldots, g_{n}\right\}$ (see Figure 3 ). The state space is $\{0,1\}^{n+1}=\left\{\left(x_{0}, \ldots, x_{n}\right) ; \forall i \in\{0, \ldots, n\}, x_{i} \in\{0,1\}\right\}$. The subgraph $T=\left(V, E^{T}\right)$ of $\mathcal{R} \mathcal{G}(S)$ with $E^{T}=\left\{\left(g_{0}, g\right) ; g \in V \backslash\left\{g_{0}\right\}\right\}$ is tree-supported (see Figure 4). From Proposition 1 and Proposition 7, there exists a symmetry of the hypercube $f$ such that $\mathcal{R G}\left(\phi_{f}(S)\right)$ is a flower-graph whose all regulations from the hub $g_{0}$ are activations.

\subsubsection{Flower-graphs: study of $\phi_{f}(S)$}

From now, we consider the FDS $\phi_{f}(S)$ and its associated flower-graph.

Let $B\left(x_{1}, \ldots, x_{n}\right)$ denote the logical rule governing $g_{0}$. We have $\phi_{f}(S)(x)=$ $\left.\left(B\left(x_{1}, \ldots, x_{n}\right), x_{0}, \ldots, x_{0}\right)\right)$, hence $\phi_{f}(S)^{2}(x)=\left(B\left(x_{0}, \ldots, x_{0}\right), a, \ldots, a\right)$ with $a=B\left(x_{1}, \ldots, x_{n}\right)$, and all the other iterates of $x$ will be of this form. This leads us to consider four cases, depending on the values of $B(0, \ldots, 0)$ and $B(1, \ldots, 1)$. For the sake of convenience, we introduce the boolean function $\tilde{B}:\{0,1\} \rightarrow\{0,1\}$ defined by $\tilde{B}(0)=B(0, \ldots, 0)$ and $\tilde{B}(1)=B(1, \ldots, 1)$.

The following proposition describes asymptotical properties of the synchronous dynamics of $\phi_{f}(S)$.

Proposition 10. The attractors of the synchronous $S T G$ of $\phi_{f}(S)$ are the following:

1. If $\tilde{B}(0)=0$ and $\tilde{B}(1)=1$, then there are two stable states $(0, \ldots, 0)$ and $(1, \ldots, 1)$ and one cyclic attractor constituted of the states $(0,1, \ldots, 1)$ and $(1,0, \ldots, 0)$.

2. If $\tilde{B}(0)=\tilde{B}(1)=0$, then there is a unique stable state $(0, \ldots, 0)$ and no cyclic attractor.

3. If $\tilde{B}(0)=\tilde{B}(1)=1$, then there is a unique stable state $(1, \ldots, 1)$ and no cyclic attractor.

4. If $\tilde{B}(0)=1$ and $\tilde{B}(1)=0$, then there is a unique cyclic attractor constituted by the states $(0, \ldots, 0),(1, \ldots, 1),(1,0, \ldots, 0)$ and $(0,1, \ldots, 1)$.

Proof. This is an easy consequence of the expression of the iterates of $\phi_{f}(S)$. 
Remark 5. Case 2 and 3 are symmetrical, by switching 0 and 1 . In other words, the action of the symmetry with respect to the center of $\mathcal{H}_{n}$ switches the second and the third cases.

We now study the asynchronous dynamics of flower-graphs, afresh using the conjugated FDS $\phi_{f}(S)$. Lemma 1 will emphasize the major role of the hub on the dynamics.

Lemma 1. Let $x$ be a state and

$$
Z_{x}=\left\{\bar{x}^{J} ; J \subset\left\{i \in\{1, \ldots, n\} ; x_{i} \neq x_{0}\right\}\right\} .
$$

We have $x \rightsquigarrow y$ for any $y \in Z_{x}$. In particular, $x \rightsquigarrow\left(x_{0}, \ldots, x_{0}\right)$.

Proof. Consider a state $x$ and $J=\left\{i_{1}, \ldots, i_{p}\right\}$ a subset of $\left\{i \in\{1, \ldots, n\} ; x_{i} \neq\right.$ $\left.x_{0}\right\}$ of cardinal $p$.

Let $x(0), \ldots, x(p)$ be the finite sequence of states defined by $x(0)=x$ and, for $k \in\{1, \ldots, p\}, x(k)=\bar{x}^{\left\{i_{1} \ldots i_{k}\right\}}$.

By Proposition 1, we know that the interaction from $g_{0}$ to $g_{i_{k}}$ belongs to the local graph $\mathcal{R G}_{x(k)}\left(\phi_{f}(S)\right)$ for any $k \in\{0, \ldots, p-1\}$. Thus $x(k) \rightsquigarrow$ $x(k+1)$, and finally $x(0) \rightsquigarrow \bar{x}^{J}=x(p)$.

The following proposition describes asymptotical properties of the asynchronous dynamics of $\phi_{f}(S)$, according to the cases described in Proposition 10.

Proposition 11. The attractors of the asynchronous $S T G$ of $\phi_{f}(S)$ are the following:

1. If $\tilde{B}(0)=0$ and $\tilde{B}(1)=1$, then there are two stable states $(0, \ldots, 0)$ and $(1, \ldots, 1)$ and no cyclic attractor.

2. If $\tilde{B}(0)=\tilde{B}(1)=0$, then there is a unique stable state $(0, \ldots, 0)$ and no cyclic attractor.

3. If $\tilde{B}(0)=\tilde{B}(1)=1$, then there is a unique stable state $(1, \ldots, 1)$ and no cyclic attractor.

4. If $\tilde{B}(0)=1$ and $\tilde{B}(1)=0$, then there is a unique attractor, which is cyclic and involves all the states.

Proof. We recall that the stable states are the same in the synchronous and asynchronous dynamics. 
1. If $\tilde{B}(0)=0$ and $\tilde{B}(1)=1$, the stable states are $(0, \ldots, 0)$ and $(1, \ldots, 1)$. The Lemma 1 shows that for any state $x$, we have $x \rightsquigarrow\left(x_{0}, \ldots, x_{0}\right)$, which is a stable state, and this implies that no state is in a cyclic attractor.

2. If $\tilde{B}(0)=\tilde{B}(1)=0$, then $(0, \ldots, 0)$ is the unique stable state. By Lemma 1 , for any state $x$ such that $x_{0}=0$, we have $x \rightsquigarrow(0, \ldots, 0)$. By the same lemma and the condition on $\tilde{B}$, if $x_{0}=1$, we have $x \rightsquigarrow(1, \ldots, 1) \rightsquigarrow(0,1, \ldots, 1) \rightsquigarrow(0, \ldots, 0)$, and no state is in a cyclic attractor.

3. The case $\tilde{B}(0)=\tilde{B}(1)=1$ is the same as the previous one, switching 0 and 1.

4. If $\tilde{B}(0)=1$ and $\tilde{B}(1)=0$, let $x$ be a state. By Lemma 1 and the condition on $\tilde{B}$, if $x_{0}=0$ we have $x \rightsquigarrow(0, \ldots, 0) \rightsquigarrow(1,0, \ldots, 0) \rightsquigarrow$ $(1, \ldots, 1) \rightsquigarrow(0,1, \ldots, 1) \rightsquigarrow x$; if $x_{0}=1$ we have the same switching 0 and 1 . Therefore the lonely attractor is cyclic, involving all the states.

Example 2. We consider particular cases, in which all the signs of the interactions of $\mathcal{R G}(S)$, and thus of $\mathcal{R} \mathcal{G}\left(\phi_{f}(S)\right)$, are determined. Let us recall that $n \geq 2$.

1. If all the petals are positive circuits, that is all the interactions of $\mathcal{R} \mathcal{G}\left(\phi_{f}(S)\right)$ are activations, then $\tilde{B}(0)=0$ and $\tilde{B}(1)=1$ (and thus two stable states and no cyclic attractor in the asynchronous dynamics).

2. If all the petals are negative circuits, that is all the interactions of $\mathcal{R G}\left(\phi_{f}(S)\right)$ towards $g_{0}$ are inhibitions, then $\tilde{B}(0)=1$ and $\tilde{B}(1)=$ 0 (and thus a unique cyclic attractor involving all the states in the asynchronous dynamics).

3. If one petal is positive and the others negative, that is for example $g_{1}$ activates $g_{0}$ and $g_{2}, \ldots, g_{n}$ inhibit $g_{0}$ in $\mathcal{R} \mathcal{G}\left(\phi_{f}(S)\right)$, we have the following alternatives:

- if $g_{0}$ admits a logical rule including the conjunctive clause $g_{1}$, then $\tilde{B}(1)=1$; otherwise $\tilde{B}(1)=0$;

- if $g_{0}$ admits a logical rule with at least one conjunctive clause including only radicals of $\left\{\neg g_{2}, \ldots, \neg g_{n}\right\}$, then $\tilde{B}(0)=1$; otherwise $\tilde{B}(0)=0$. 
Let us then remark that $\tilde{B}(1)=1$ implies $\tilde{B}(0)=1$, leaving the three effective possible cases $\tilde{B}(0)=\tilde{B}(1)=1, \tilde{B}(0)=\tilde{B}(1)=0, \tilde{B}(0)=1$ and $\tilde{B}(1)=0$ :

- the case $\tilde{B}(0)=\tilde{B}(1)=1$ (and thus one stable state and no cyclic attractor in the asynchronous dynamics) is the case where $g_{1}$ does not need to cooperate with other regulators to regulate the hub and where $g_{2}, \ldots, g_{n}$ do not need to cooperate with $g_{1}$ to regulate the hub;

- the case $\tilde{B}(0)=\tilde{B}(1)=0$ (and thus one stable state and no cyclic attractor in the asynchronous dynamics) is the case where $g_{1}$ and components among $g_{2}, \ldots, g_{n}$ need always to cooperate to regulate the hub;

- the case $\tilde{B}(0)=1$ and $\tilde{B}(1)=0$ (and thus one cyclic attractor involving all the states in the asynchronous dynamics) is the case where $g_{1}$ needs to cooperate with other regulators to regulate the hub and where some components among $g_{2}, \ldots, g_{n}$ do not need to cooperate with $g_{1}$ to regulate the hub.

4. If one petal is negative and the others positive, that is for example $g_{1}$ inhibits $g_{0}$ and $g_{2}, \ldots, g_{n}$ activ $g_{0}$ in $\mathcal{R G}\left(\phi_{f}(S)\right)$, we have the two following alternatives:

- if $g_{0}$ admits a logical rule including the conjunctive clause $\neg g_{1}$, then $\tilde{B}(0)=1$; otherwise $\tilde{B}(0)=0$;

- if $g_{0}$ admits a logical rule with at least one conjunctive clause including only radicals of $\left\{g_{2}, \ldots, g_{n}\right\}$, then $\tilde{B}(1)=1$; otherwise $\tilde{B}(1)=0$.

Let us then remark that $\tilde{B}(0)=1$ implies $\tilde{B}(1)=1$, leaving the three effective possible cases $\tilde{B}(0)=\tilde{B}(1)=1, \tilde{B}(0)=\tilde{B}(1)=0, \tilde{B}(0)=0$ and $\tilde{B}(1)=1$ :

- the case $\tilde{B}(0)=\tilde{B}(1)=1$ (and thus one stable state and no cyclic attractor in the asynchronous dynamics) is the case where $g_{1}$ does not need to cooperate with other regulators to regulate the hub and where $g_{2}, \ldots, g_{n}$ do not need to cooperate with $g_{1}$ to regulate the hub;

- the case $\tilde{B}(0)=\tilde{B}(1)=0$ (and thus one stable state and no cyclic attractor in the asynchronous dynamics) is the case where $g_{1}$ and components among $g_{2}, \ldots, g_{n}$ need always to cooperate to regulate the hub;

- the case $\tilde{B}(0)=0$ and $\tilde{B}(1)=1$ (and thus two stable states and no cyclic attractor in the asynchronous dynamics) is the case where $g_{1}$ needs to cooperate with other regulators to regulate the hub and where 
some components among $g_{2}, \ldots, g_{n}$ do not need to cooperate with $g_{1}$ to regulate the hub.

\subsubsection{Flower-graphs: general case}

We have the following theorems as immediate corollary of the study of $\phi_{f}(S)$.

Theorem 1. Let $S$ be a FDS whose associated regulatory graph $\mathcal{R G}(S)$ is a flower-graph. Then $S$ has zero, one or two stable state(s), and the attractors of the synchronous dynamics are the following:

1. If $S$ has two stable states, then the attractors are these states and one cyclic attractor including two states.

2. If $S$ has one stable state, it is the unique attractor.

3. If $S$ has no stable state, then there is a unique cyclic attractor including four states.

Theorem 2. Let $S$ be a FDS whose associated regulatory graph $\mathcal{R} \mathcal{G}(S)$ is a flower-graph.

1. If $S$ has one or two stable state(s), then they are the lonely attractors of the asynchronous dynamics.

2. If $S$ has no stable state, then the lonely attractor of the asynchronous dynamics is cyclic and involves all the states.

Moreover the following proposition derived from Example 2 precises a result of [3].

Proposition 12. Let $S$ be a FDS whose associated regulatory graph $\mathcal{R G}(S)$ is a flower-graph whose signs of interactions are determined.

1. If all the petals are positive circuits, then $S$ has two stable states which are the lonely attractors of the asynchronous dynamics.

2. If all the petals are negative circuits, then the lonely attractor of the asynchronous dynamics is cyclic and involves all the states.

3. If one petal is positive and the others negative, then either $S$ has one stable state which is the lonely attractor of the asynchronous dynamics, or the lonely attractor is cyclic and involves all the states. 
4. If one petal is negative and the others positive, then $S$ has one or two stable state(s), that are the lonely attractors of the asynchronous dynamics.

Remark 6. Example 2 allows to precise Items 3 and 4:

- In Item 3, if the positive petal is related to the component $g_{1}$, then one has a cyclic attractor if and only if the hub admits a logical rule without the conjunctive clauses $g_{1}$ and $\neg g_{1}$, and at least one conjunctive clause with neither the radical $g_{1}$ nor $\neg g_{1}$. In other words, one has a cyclic attractor if and only if $g_{1}$ needs to cooperate with other regulators to regulate the hub and where some components among $g_{2}, \ldots, g_{n}$ do not need to cooperate with $g_{1}$ to regulate the hub.

- In Item 4 , if the negative petal is related to a component $g_{1}$, then one has two stable states if and only if the hub admits a logical rule without the conjunctive clauses $g_{1}$ and $\neg g_{1}$, and at least one conjunctive clause with neither the radical $g_{1}$ nor $\neg g_{1}$. In other words, one has two stable states if and only if $g_{1}$ needs to cooperate with other regulators to regulate the hub and where some components among $g_{2}, \ldots, g_{n}$ do not need to cooperate with $g_{1}$ to regulate the hub.

Remark 7. In the case of the flower-graphs, it is not simple to enumerate the classes of isometric FDS; indeed the previous study focuses on four values taken by $B$, letting a lot of degrees of freedom for other values. We can assert that the classes of FDS whose associated $R G$ are flower-graphs spread into three families of classes: classes of FDS with zero stable state, with one stable state, with two stable states. Moreover we have seen that each class contains elements on the form $\left(x_{0}, \ldots x_{n}\right) \mapsto\left(B\left(x_{1}, \ldots, x_{n}\right), x_{0}, \ldots, x_{0}\right)$, and therefore the number of classes is less than the number of functions $B$ depending actually on each coordinate $x_{1}, \ldots, x_{n}$.

Example 3. As an illustration of the above, we detail the case $n=2$.

Each class contains elements on the form $S_{B}:\left(x_{0}, x_{1}, x_{2}\right) \mapsto\left(B\left(x_{1}, x_{2}\right), x_{0}, x_{0}\right)$, where $B\left(x_{1}, x_{2}\right)$ is given by one of the 10 possibilities $B\left(x_{1}, x_{2}\right)=x_{1} \wedge x_{2}$, $x_{1} \wedge \neg x_{2}, \neg x_{1} \wedge x_{2}, \neg x_{1} \wedge \neg x_{2}, x_{1} \vee x_{2}, x_{1} \vee \neg x_{2}, \neg x_{1} \vee x_{2}, \neg x_{1} \vee \neg x_{2}$, $x_{1} \oplus x_{2}, x_{1} \oplus \neg x_{2}$ (see Remark 4).

Given $B$ and an isometry $f$ of the cube, it is easy to verificate that $\phi_{f}(S)$ is on the form $S_{B^{\prime}}\left(x_{0}, x_{1}, x_{2}\right)=\left(B^{\prime}\left(x_{1}, x_{2}\right), x_{0}, x_{0}\right)$ if and only if $f\left(x_{0}, x_{1}, x_{2}\right)=\left(x_{0}, x_{1}, x_{2}\right)$ or $\left(\overline{x_{0}}, \overline{x_{1}}, \overline{x_{2}}\right)$ or $\left(x_{0}, x_{2}, x_{1}\right)$ or $\left(\overline{x_{0}}, \overline{x_{2}}, \overline{x_{1}}\right)$.

In other words, $S_{B}$ and $S_{B^{\prime}}$ are isometric if and only $B^{\prime}\left(x_{1}, x_{2}\right)=$ $B\left(x_{1}, x_{2}\right)$, or $\neg B\left(\neg x_{1}, \neg x_{2}\right)$, or $B\left(x_{2}, x_{1}\right)$, or $\neg B\left(\neg x_{2}, \neg x_{1}\right)$. 
Hence, there exists four classes of isometric boolean FDS whose RG are flower-graphs with two petals:

- a class containing $S_{B}$ for $B\left(x_{1}, x_{2}\right)=x_{1} \wedge x_{2}$ and $B\left(x_{1}, x_{2}\right)=x_{1} \vee x_{2}$,

- a class containing $S_{B}$ for $B\left(x_{1}, x_{2}\right)=x_{1} \wedge \neg x_{2}, B\left(x_{1}, x_{2}\right)=\neg x_{1} \wedge x_{2}$, $B\left(x_{1}, x_{2}\right)=x_{1} \vee \neg x_{2}$ and $B\left(x_{1}, x_{2}\right)=\neg x_{1} \vee x_{2}$,

- a class containing $S_{B}$ for $B\left(x_{1}, x_{2}\right)=\neg x_{1} \wedge \neg x_{2}$ and $B\left(x_{1}, x_{2}\right)=$

$\neg x_{1} \vee \neg x_{2}$,

- a class containing $S_{B}$ for $B\left(x_{1}, x_{2}\right)=x_{1} \oplus x_{2}$ and $B\left(x_{1}, x_{2}\right)=x_{1} \oplus \neg x_{2}$.

\subsection{Extensions to hub-graphs}

We now study boolean FDS whose RG are hub-graphs, that are directed graphs $(V, E)$ such that there exists a particular node $g_{0} \in V$ called hub satisfying the following properties: the hub $g_{0}$ is the only vertex possibly submitted to more than one regulations, $\left(g_{0}, g_{0}\right) \notin E$, and for any vertex $g$, one has $g_{0} \rightsquigarrow g$ in the graph (see Figure 3 ).

Remark that isolated circuits, chorded circuits without loops and flowergraphs are hub-graphs.

Let $S$ be a boolean FDS whose associated regulatory graph $\mathcal{R G}(S)$ is a hub-graph, with hub $g_{0}$ and $V=\left\{g_{0}, \ldots, g_{n}\right\}$. The state space is $\{0,1\}^{n+1}=$ $\left\{\left(x_{0}, \ldots, x_{n}\right) ; \forall i \in\{0, \ldots, n\}, x_{i} \in\{0,1\}\right\}$.

Remark 8. The definition of a hub-graph implies that no component is self-regulated in $\mathcal{R G}(S)$ : it is provided for the hub in the definition, and if $g \neq g_{0}$, a self-regulation on $g$ would make it regulated by itself and by another component in a path from $g_{0}$ to $g$.

Lemma 2. The subgraph $T=\left(V, E^{T}\right)$ of $\mathcal{R} \mathcal{G}(S)$ with $E^{T}=E \backslash\left\{\left(g, g_{0}\right) \in E\right\}$ is tree-supported.

Proof. First of all, the undirected graph $\tilde{T}=\left(V,\left\{\{a, b\} ;(a, b) \in E^{T}\right.\right.$ and $a \neq$ $b\})$ is connected, since for any component $g \neq g_{0}$, we have $g_{0} \rightsquigarrow g$ in $\mathcal{R G}(S)$. In addition, if there exists a cycle in $\tilde{T}$, the hub is not a vertex of this cycle by definition of $T$. Moreover this cycle corresponds to a circuit in $T$, since otherwise one component of the cycle, different of the hub, would have at least two regulators, vertices of the cycle; the existence of a cycle in $\tilde{T}$ is thus impossible and $\tilde{T}$ is connected and acyclic: $\tilde{T}$ is a tree.

In a similar way, $T$ does not include both an edge $(a, b)$ and its reverse $(b, a)$ (same argument as Remark 8). In conclusion, $T$ is tree-supported. 
Accordingly, from Proposition 1 and Proposition 7, there exists a symmetry of the hypercube $f$ such that $\mathcal{R G}\left(\phi_{f}(S)\right)$ is a hub-graph whose all regulations which are not towards the hub $g_{0}$ are activations (see Figure 4 ).

\subsubsection{Hub-graphs: study of $\phi_{f}(S)$}

In the following, we denote by $T_{f}$ the image in $\mathcal{R G}\left(\phi_{f}(S)\right)$ of the subgraph $T$ of $\mathcal{R G}(S)$.

The set of vertices of $\mathcal{R G}(S)$ (components) can be partitioned according to the graph distance to the hub in $T_{f}$, and we order the components according to this distance. For the sake of convenience, we renumber the components

$$
g_{0} ; g_{1,1}, \ldots, g_{1, r_{1}} ; \ldots ; g_{d, 1}, \ldots, g_{d, r_{d}},
$$

where $d$ is the maximal distance to the hub in $T_{f}$ (hence, the distance of $g_{1,1}, \ldots, g_{1, r_{1}}$ to $g_{0}$ is equal to 1 , and so on),

and the coordinates of a state

$$
x=\left(x_{0} ; x_{1,1}, \ldots, x_{1, r_{1}} ; \ldots ; x_{d, 1}, \ldots, x_{d, r_{d}}\right) .
$$

Then there exists a boolean function $B:\{0,1\}^{n-1} \rightarrow\{0,1\}$ such that

$\phi_{f}(S)(x)=\left(B\left(x_{1,1}, \ldots, x_{1, r_{1}} ; \ldots ; x_{d, 1}, \ldots, x_{d, r_{d}}\right) ; x_{0}, \ldots, x_{0} ; x_{1,1}, \ldots, x_{1, r_{1}} ; \ldots ; x_{d-1,1}, \ldots, x_{d-1, r_{d-1}}\right)$.

due to the fact that there are only activations on $T_{f}$. Of course, the function $B$ depends effectively only on the $x_{j}$ related to regulators of $g_{0}$.

The result is that for $k \geq d+1$ the iterates $\phi_{f}(S)^{k}(x)$ of a state $x$ are of the form

$$
\phi_{f}(S)^{k}(x)=\left(a_{0} ; a_{1}, \ldots, a_{1} ; \ldots ; a_{d}, \ldots, a_{d}\right),
$$

and the boolean function $\tilde{B}:\{0,1\}^{d} \rightarrow\{0,1\}$ defined by $\tilde{B}\left(a_{1}, \ldots, a_{d}\right)=$ $B\left(a_{1}, \ldots, a_{1} ; \ldots ; a_{d}, \ldots, a_{d}\right)$ gives

$$
\phi_{f}(S)^{k+1}(x)=\left(\tilde{B}\left(a_{1}, \ldots, a_{d}\right) ; a_{0}, \ldots, a_{0} ; \ldots ; a_{d-1}, \ldots, a_{d-1}\right) .
$$

This allows to determine the stable states of $\phi_{f}(S)$.

Proposition 13. The stable states of $\phi_{f}(S)$ are the following:

1. If $\tilde{B}(0, \ldots, 0)=0$ and $\tilde{B}(1, \ldots, 1)=1$, then there are two stable states $(0, \ldots, 0)$ and $(1, \ldots, 1)$. 
2. If $\tilde{B}(0, \ldots, 0)=\tilde{B}(1, \ldots, 1)=0$, then there is a unique stable state $(0, \ldots, 0)$.

3. If $\tilde{B}(0, \ldots, 0)=\tilde{B}(1, \ldots, 1)=1$, then there is a unique stable state $(1, \ldots, 1)$.

4. If $\tilde{B}(0, \ldots, 0)=1$ and $\tilde{B}(1, \ldots, 1)=0$, then there is no stable state.

Proof. This is an immediate consequence of the expression of the iterates of $\phi_{f}(S)$.

The following lemma generalizes a part of Lemma 1 on the asynchronous dynamics of flower-graphs to hub-graphs.

Lemma 3. Let $x$ be a state. In $\mathcal{G}_{a}\left(\phi_{f}(S)\right)$, one has $x \rightsquigarrow\left(x_{0}, \ldots, x_{0}\right)$.

Proof. Consider a state $x=\left(x_{0} ; x_{1,1}, \ldots, x_{1, r_{1}} ; \ldots ; x_{d, 1}, \ldots, x_{d, r_{d}}\right)=$ $\left(x_{0}, \ldots, x_{n}\right)$. All regulations which are not towards the hub $g_{0}$ being activations, the ordering of the coordinates of $x$ shows that we have $x \rightsquigarrow$ $\left(x_{0}, x_{0}, x_{2}, \ldots, x_{n}\right) \rightsquigarrow\left(x_{0}, x_{0}, x_{0}, x_{3}, \ldots, x_{n}\right)$, and so on until $x \rightsquigarrow\left(x_{0}, \ldots, x_{0}\right)$.

It is now easy to describe the asymptotic asynchronous dynamics of $\phi_{f}(S)$.

Proposition 14. The attractors of the asynchronous $S T G$ of $\phi_{f}(S)$ are the following:

1. If $\tilde{B}(0, \ldots, 0)=0$ and $\tilde{B}(1, \ldots, 1)=1$, then there are two stable states $(0, \ldots, 0)$ and $(1, \ldots, 1)$ and no cyclic attractor.

2. If $\tilde{B}(0, \ldots, 0)=\tilde{B}(1, \ldots, 1)=0$, then there is a unique stable state $(0, \ldots, 0)$ and no cyclic attractor.

3. If $\tilde{B}(0, \ldots, 0)=\tilde{B}(1, \ldots, 1)=1$, then there is a unique stable state $(1, \ldots, 1)$ and no cyclic attractor.

4. If $\tilde{B}(0, \ldots, 0)=1$ and $\tilde{B}(1, \ldots, 1)=0$, then there is a unique cyclic attractor and no stable state.

Proof. The proofs of the cases 1, 2 and 3 are exactely the same as in Proposition 11 .

If $\tilde{B}(0, \ldots, 0)=1$ and $\tilde{B}(1, \ldots, 1)=0$, let $x$ be a state. By Lemma 3 and the condition on $\tilde{B}$, if $x_{0}=0$ we have $x \rightsquigarrow(0, \ldots, 0) \rightsquigarrow(1,0, \ldots, 0) \rightsquigarrow$ $(1, \ldots, 1)$; if $x_{0}=1$ we have the same switching 0 and 1 . Therefore the lonely attractor is cyclic. 
Example 4. Extending Example 2, we consider particular cases in which all the signs of the circuits of $\mathcal{R G}(S)$, and thus of $\mathcal{R G}\left(\phi_{f}(S)\right)$, are determined.

1. If all the circuits are positive, that is all the interactions of $\mathcal{R} \mathcal{G}\left(\phi_{f}(S)\right)$ towards $g_{0}$ are activations, then $\tilde{B}(0, \ldots, 0)=0$ and $\tilde{B}(1, \ldots, 1)=1$ (and thus two stable states and no cyclic attractor in the asynchronous dynamics).

2. If all the circuits are negative circuits, that is all the interactions of $\mathcal{R G}\left(\phi_{f}(S)\right)$ towards $g_{0}$ are inhibitions, then $\tilde{B}(0, \ldots, 0)=1$ and $\tilde{B}(1, \ldots, 1)=0$ (and thus a unique cyclic attractor and no stable state in the asynchronous dynamics).

3. If there is at least two circuits, one positive and the others negative, that is some component $g$ activs $g_{0}$ and the other regulators of $g_{0}$ are inhibitors in $\mathcal{R G}\left(\phi_{f}(S)\right)$, we have the two following alternatives:

- if $g_{0}$ admits a logical rule including the conjunctive clause $g$, then $\tilde{B}(1, \ldots, 1)=1$; otherwise $\tilde{B}(1, \ldots, 1)=0$;

- if $g_{0}$ admits a logical rule with at least one conjunctive clause including only regulators $\neq g$, then $\tilde{B}(0, \ldots, 0)=1$; otherwise $\tilde{B}(0, \ldots, 0)=$ 0 .

Let us then remark that $\tilde{B}(1, \ldots, 1)=1$ implies $\tilde{B}(0, \ldots, 0)=1$, leaving the three effective possible cases $\tilde{B}(0, \ldots, 0)=\tilde{B}(1, \ldots, 1)=1$, $\tilde{B}(0, \ldots, 0)=\tilde{B}(1, \ldots, 1)=0, \tilde{B}(0, \ldots, 0)=1$ and $\tilde{B}(1, \ldots, 1)=0$ :

- the case $\tilde{B}(0, \ldots, 0)=\tilde{B}(1, \ldots, 1)=1$ (and thus one stable state and no cyclic attractor in the asynchronous dynamics) is the case where $g$ does not need to cooperate with other regulators to regulate the hub and where the other regulators do not need to cooperate with $g$ to regulate the hub;

- the case $\tilde{B}(0, \ldots, 0)=\tilde{B}(1, \ldots, 1)=0$ (and thus one stable state and no cyclic attractor in the asynchronous dynamics) is the case where $g$ and other regulators need always to cooperate to regulate the hub;

- the case $\tilde{B}(0, \ldots, 0)=1$ and $\tilde{B}(1, \ldots, 1)=0$ (and thus a unique cyclic attractor and no stable state in the asynchronous dynamics) is the case where $g$ needs to cooperate with other regulators to regulate the hub and where some other regulators do not need to cooperate with $g$ to regulate the hub. 
4. If there is at least two circuits, one negative and the others positive, that is some component $g$ inhibits $g_{0}$ and the other regulators of $g_{0}$ are activators in $\mathcal{R G}\left(\phi_{f}(S)\right)$, we have the following alternatives:

- if $g_{0}$ admits a logical rule including the conjunctive clause $\neg g$, then $\tilde{B}(0, \ldots, 0)=1$; otherwise $\tilde{B}(0, \ldots, 0)=0$;

- if $g_{0}$ admits a logical rule with at least one conjunctive clause including only regulators $\neq g$, then $\tilde{B}(1, \ldots, 1)=1$; otherwise $\tilde{B}(1, \ldots, 1)=$ 0 .

Let us then remark that $\tilde{B}(0, \ldots, 0)=1$ implies $\tilde{B}(1, \ldots, 1)=1$, leaving the three effective possible cases $\tilde{B}(0, \ldots, 0)=\tilde{B}(1, \ldots, 1)=1$, $\tilde{B}(0, \ldots, 0)=\tilde{B}(1, \ldots, 1)=0, \tilde{B}(0, \ldots, 0)=0$ and $\tilde{B}(1, \ldots, 1)=1$ :

- the case $\tilde{B(0}, \ldots, 0)=\tilde{B(1}, \ldots, 1)=1$ (and thus one stable state and no cyclic attractor in the asynchronous dynamics) is the case where $g$ does not need to cooperate with other regulators to regulate the hub and where the other regulators do not need to cooperate with $g$ to regulate the hub;

- the case $\tilde{B}(0, \ldots, 0)=\tilde{(} 1, \ldots, 1)=0$ (and thus one stable state and no cyclic attractor in the asynchronous dynamics) is the case where $g$ and other regulators need always to cooperate to regulate the hub;

- the case $\tilde{B}(0, \ldots, 0)=0$ and $\tilde{B}(1, \ldots, 1)=1$ (and thus two stable states and no cyclic attractor in the asynchronous dynamics) is the case where $g$ needs to cooperate with other regulators to regulate the hub and where some other regulators do not need to cooperate with $g$ to regulate the hub.

\subsubsection{Hub-graphs: general case}

In the general case of hub-graphs, it is not possible to get a simple description of the cyclic attractors of the synchronous dynamics: there is a large number of possibilities, related to the degrees of freedom on the function $\tilde{B}$ introduced in the previous paragraph. This is illustrated by the cases of isolated circuits, chorded circuits and flower-graphs ([11] and Example 3).

The following theorem is an immediate consequence of the study of $\phi_{f}(S)$.

Theorem 3. Let $S$ be a FDS whose associated regulatory graph $\mathcal{R G}(S)$ is a hub-graph. Then $S$ has zero, one or two stable state(s). 
1. If $S$ has one or two stable state(s), then they are the lonely attractors of the asynchronous dynamics.

2. If $S$ has no stable state, then the lonely attractor of the asynchronous dynamics is cyclic.

Remark 9. - The study of $\phi_{f}(S)$ has proved that the computation of two values of the logical rule of the hub allows to determine the type of asymptotic behavior of the asynchronous dynamics of $S$, among the three possibilities given by the theorem. Indeed, if, in practice, going through $\phi_{f}(S)$ and computing $\tilde{B}(0, \ldots, 0)=1$ and $\tilde{B}(1, \ldots, 1)=0$ is the easiest way to conclude, this can be considered as the computation of two values of the logical formula of the hub, coming back to $S$ via $f^{-1}$.

- Moreover this may be interpreted in depth in terms of cooperation or non cooperation between the regulators of the hub, as it is detailed in particular cases below.

The following proposition derived from Example 4 is an extension of Proposition 12 precises a result of [3].

Proposition 15. Let $S$ be a FDS whose associated regulatory graph $\mathcal{R G}(S)$ is a hub-graph whose signs of the circuits are determined.

1. If all the circuits are positive circuits, then $S$ has two stable states which are the lonely attractors of the asynchronous dynamics.

2. If all the circuits are negative circuits, then the lonely attractor of the asynchronous dynamics is cyclic.

3. If there is at least two circuits, one positive and the others negative, then either $S$ has one stable state which is the lonely attractor of the asynchronous dynamics, or the lonely attractor is cyclic.

4. If there is at least two circuits, one negative and the others positive, then $S$ has one or two stable state(s), that are the lonely attractors of the asynchronous dynamics.

Remark 10. Example 4 allows to precise Items 3 and 4:

- In Item 3, if the positive circuit is related to a regulator $g$ of $g_{0}$, then one has a cyclic attractor if and only if the hub admits a logical rule without the conjunctive clauses $g$ and $\neg g$, and at least one conjunctive clause without the radicals $g$ and $\neg g$. In other words, one has a cyclic attractor if and only 
if $g$ needs to cooperate with other regulators to regulate the hub and where some other regulators do not need to cooperate with $g$ to regulate the hub.

- In Item 4, if the negative circuit is related to a regulator $g$ of $g_{0}$, then one has two stable states if and only if the hub admits a logical rule without the conjunctive clauses $g$ and $\neg g$ and at least one conjunctive clause without the radicals $g$ and $\neg g$. In other words, one has two stable states if and only if $g$ needs to cooperate with other regulators to regulate the hub and where some other regulators do not need to cooperate with $g$ to regulate the hub.

\section{Application to the negative Thomas's rule}

In the boolean context, the negative Thomas's rule can be written in the following terms:

"Given $S: X \rightarrow X$ a boolean FDS such that the asynchronous dynamics of $S$ displays a cyclic attractor, then the regulatory graph of $S$ contains a negative circuit."

Furthermore in case of the existence of a cyclic attractor, several examples show that it is possible that no local graph $\mathcal{R} \mathcal{G}_{x}(S)$ including a negative circuit can be found [16]. This stresses the fact that a fine understanding of the interpretation of this rule in terms of local and hence functional interactions has to be specified.

"Given $S: X \rightarrow X$ a boolean FDS such that the asynchronous dynamics of $S$ contains a cyclic attractor, there exists a circuit of $\mathcal{R} \mathcal{G}(S)$, and for each interaction of this circuit a pair of states on the form $\left\{x, \bar{x}^{i}\right\}$ where this interaction is expressed, such that the product of the related signs is negative."

We are going to give a new proof of this rule in the light of the action of the symmetries of the hypercube.

Remark 11. If $\mathcal{R G}(S)$ contains a circuit such that at least one of its interaction has an indeterminated sign (i.e. sign depending on the pairs of states where it is expressed), this implies the presence of a negative circuit. For this reason, we will suppose that all the signs of the interactions of the circuits of $\mathcal{R} \mathcal{G}(S)$ are determined.

Notations 3. Let $x$ a state and $a \in\{0,1\}$,

$$
U_{p d}(x, a)=\left\{i \in U p d_{S}(x) ; x_{i}=a\right\} .
$$


Lemma 4. Let $S: X \rightarrow X$ be a boolean FDS such that all the interactions of $\mathcal{R G}(S)$ are activations. Then for any state $x$, there exists a stable state $y$ such that $x \rightsquigarrow y$ in $\mathcal{G}_{a}(S)$.

Proof. Let $x$ be a state such that $S(x) \neq x$.

- If $U p d_{S}(x, 0)=\emptyset$, then set $z=x$. Otherwise, suppose that $U p d_{S}(x, 0) \neq$ $\emptyset$. There exists a path $\left(x=x^{(0)}, \ldots, x^{(r)}=z\right)$ in $\mathcal{G}_{a}(S)$ such that, for $0 \leq k \leq r-1, x^{(k+1)}={\overline{x^{(k)}}}^{i_{k}}$, with $i_{k} \in U p d_{S}\left(x^{(k)}, 0\right)$ and $\operatorname{Upd}_{S}(z, 0)=\emptyset$. Indeed, at every step of building $x^{(k+1)}$ from $x^{(k)}$ one coordinate is switched from 0 to 1 , so that the process is required to end at a state $z$.

- If $U p d_{S}(z, 1)=\emptyset$, then set $y=z$. The state $y$ is a stable state and $x \rightsquigarrow y$ in $\mathcal{G}_{a}(S)$.

- If $U p d_{S}(z, 1) \neq \emptyset$, there exists a path $\left(z=z^{(0)}, \ldots, z^{(s)}=y\right)$ in $\mathcal{G}_{a}(S)$ such that, for $0 \leq k \leq s-1, z^{(k+1)}={\overline{z^{(k)}}}^{j_{k}}$, with $j_{k} \in U p d_{S}\left(z^{(k)}, 1\right)$ and $U p d_{S}(y, 1)=\emptyset$. Such a path exists since at every step of building $z^{(k+1)}$ from $z^{(k)}$ one coordinate is switched from 1 to 0 . Moreover, the hypothesis on $S$ (all interactions are activations) implies that $U p d_{S}\left(z^{(0)}, 0\right)=\cdots=$ $\operatorname{Upd}_{S}\left(z^{(s)}, 0\right)=\emptyset$. Thus $x \rightsquigarrow y$ in $\mathcal{G}_{a}(S)$, and the equalities $\operatorname{Upd}_{S}(y, 1)=$ $U p d_{S}(y, 0)=\emptyset$ mean that $y$ is a stable state.

Lemma 5. Let $S: X \rightarrow X$ be a boolean FDS such that $\mathcal{R G}(S)$ is strongly connected, all the signs of the interactions are determined, and all the circuits are positive. There exists a symmetry $f \in \Gamma\left(\mathcal{H}_{n}\right)$ such that all the interactions of $\mathcal{R G}(S)$ become activations under the conjugation by $f$.

Proof. The graph $\mathcal{R} \mathcal{G}(S)$ being strongly connected, let us consider a circuit in this graph involving all the components, that we can write after their potential renumbering

$$
C=\left(g_{1} g_{2} X_{2} g_{3} X_{3} \ldots g_{n} X_{n}\right),
$$

where $X_{k}$ is a sequence of components either empty, or of the form $Y_{k} g_{i_{k}}$ with $Y_{k}$ possibly empty and all the components of $X_{k}$ having indices $<k$.

Let us consider the subgraph $T=(V, E)$ of $\mathcal{R G}(S)$, where the set $V$ is the set of all the components, and $E$ is composed by

- the interactions of $C$ of the form $\left(g_{k}, g_{k+1}\right)$, with $k \leq n-1$, for which $X_{k}$ is empty,

- the interactions $\left(g_{i k}, g_{k+1}\right)$, with $k \leq n-1$, for which $X_{k}$ is not empty.

The subgraph $T$ is tree-supported (see section 3.4, and an example in Figure 5). By Proposition 7 and the hypothesis on the determination of 


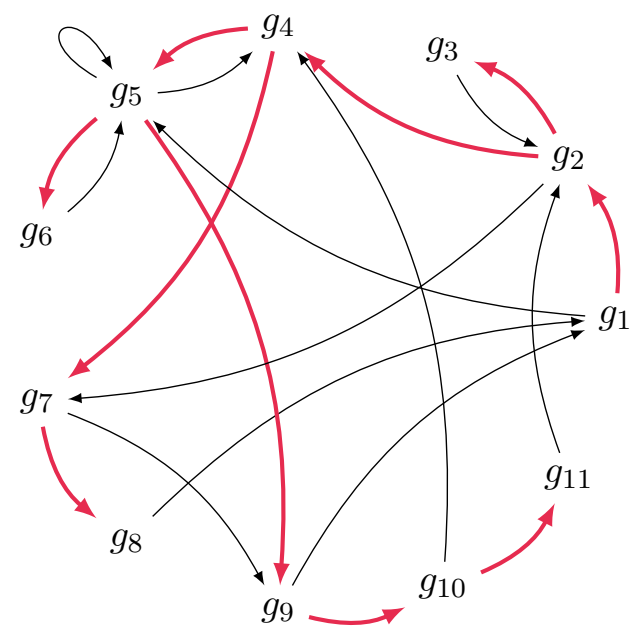

Figure 5: Illustration of Lemma 5's proof with

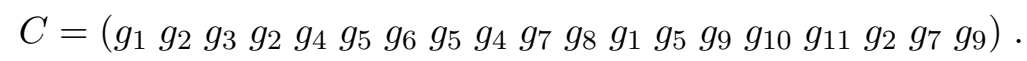

the signs, there exists a symmetry $f \in \Gamma\left(\mathcal{H}_{n}\right)$ such that all the interactions of $T$ become activations under the conjugation by $f$. Let us consider the interactions of $C$ in order of appearance: the action of $f$ preserving signs of circuits (cf. Corollary 1), and by the hypothesis on the signs of the circuits of $\mathcal{R G}(S)$, it turns out that these interactions become activations under the conjugation by $f$. It is the same for the loops of $\mathcal{R G}(S)$, and for the remaining interactions of $\mathcal{R} \mathcal{G}(S)$, that we can include in a circuit whose other interactions are in $C$ : all the interactions of $\mathcal{G}_{a}\left(\phi_{f}(S)\right)$ are activations.

Corollary 2. Let $S: X \rightarrow X$ be a boolean FDS such that $\mathcal{R} \mathcal{G}(S)$ is strongly connected, all the signs of the interactions are determined, and all the circuits are positive. Then for any state $x$, there exists a stable state $y$ such that $x \rightsquigarrow y$ in $\mathcal{G}_{a}(S)$.

Proof. Lemmas 4 and 5 give that for any state $x$, there exists a stable state $y$ such that $x \rightsquigarrow y$ in $\mathcal{G}_{a}\left(\phi_{f}(S)\right)$, and hence the same for $\mathcal{G}_{a}(S)$.

It is well-known that the strongly connected components of a directed graph $G$ are the vertices of an acyclic directed graph $G_{\mathcal{S C C}}$. Therefore, 
there exists a numbering of these components $\mathcal{C}_{1}, \ldots, \mathcal{C}_{m}$ giving a topological sorting: if there is a path from $\mathcal{C}_{k}$ to $\mathcal{C}_{h}$ in $G_{\mathcal{S C C}}$, then $k<h[19,1]$.

Theorem 4. - equivalent to the negative Thomas'rule -

Let $S: X \rightarrow X$ be a boolean FDS such that all the signs of the interactions of the circuits of $\mathcal{R} \mathcal{G}(S)$ are determined, and all these circuits are positive. Then $\mathcal{G}_{a}(S)$ does not include any cyclic attractor.

Proof. Let $\mathcal{C}_{1}, \ldots, \mathcal{C}_{m}$ be the strongly connected components of $\mathcal{R} \mathcal{G}(S)$, numbered by a topological sorting of their acyclic graph, and, for $i \in\{1, \ldots, m\}$, let $\mathcal{V}_{i}$ be the set of indices of the vertices of $\mathcal{C}_{i}$.

Using the ingredients of Lemma 5 and Proposition 7, for each $i \in$ $\{1, \ldots, m\}$, there exists a symmetry $f_{i} \in \Gamma\left(\mathcal{H}_{n}\right)$, product of hyperplane symmetries, such that all the interactions of $\mathcal{C}_{i}$ become activations under the conjugation by $f_{i}$, and such that $\left\{k \in\{1, \ldots, n\} ; \vec{f}_{i}\left(\overrightarrow{e_{k}}\right)=-\overrightarrow{e_{k}}\right\} \subset \mathcal{V}_{i}$. Let $f$ be the commutative product of the $f_{i}$. Then the strongly connected components of the conjugated regulatory graph $\mathcal{R} \mathcal{G}\left(\phi_{f}(S)\right)$ are $\mathcal{C}_{1}, \ldots, \mathcal{C}_{m}$, all the signs of the interactions of the circuits of $\mathcal{R G}\left(\phi_{f}(S)\right)$ are determined, and all these interactions are activations.

Let $x$ be a state. Lemma 4 allows to update the coordinates $x_{i}$ of $x$ related to the $g_{i}$ which are components of $\mathcal{C}_{1}$, in other words such that $i \in \mathcal{V}_{1}$; this is achieved through a path $x \rightsquigarrow x^{j_{1}}$ of $\mathcal{G}_{a}\left(\phi_{f}(S)\right)$ such that if $x^{j_{1}} \rightsquigarrow z$ in $\mathcal{G}_{a}\left(\phi_{f}(S)\right)$, then $U p d_{\phi_{f}(S)}(z)$ does not contain any integer of $\mathcal{V}_{1}$, because of the way the $\mathcal{C}_{i}$ were numbered.

If $m>1$, we have now to consider the interactions of components of $\mathcal{C}_{1}$ on components of $\mathcal{C}_{2}$. The coordinates of $x$ related to $\mathcal{V}_{1}$ being definitively stabilized, we consider that they are inputs for $\mathcal{C}_{2}$, and we can rewrite the logical formulas for the components of $\mathcal{C}_{2}$, taking into account these inputs. Some interactions of $\mathcal{C}_{2}$ may disappear, but all the remaining ones will be activations.

Then Lemma 4 allows to update the coordinates $x_{i}$ of $x$ such that $i \in \mathcal{V}_{2}$; this is achieved through a path $x \rightsquigarrow x^{j_{1}} \rightsquigarrow x^{j_{2}}$ of $\mathcal{G}_{a}\left(\phi_{f}((S))\right.$ such that if $x^{j_{2}} \rightsquigarrow z$ in $\mathcal{G}_{a}\left(\phi_{f}((S))\right.$, then $\operatorname{Upd}_{\phi_{f}(S)}(z)$ does not contain any integer of $\mathcal{V}_{1} \cup \mathcal{V}_{2}$

If $m>2$, we consider the interactions of components of $\mathcal{C}_{1}$ and $\mathcal{C}_{2}$ on components of $\mathcal{C}_{3}$, and so on.

At the end of the process, we obtain a path

$$
x \rightsquigarrow x^{j_{1}} \rightsquigarrow \cdots \rightsquigarrow x^{j_{m}}=y
$$


in $\mathcal{G}_{a}\left(\phi_{f}((S))\right.$ such that if $k \in\{1, \ldots, m\}$ and $x^{j_{k}} \rightsquigarrow z$ in $\mathcal{G}_{a}\left(\phi_{f}((S))\right.$, then $U p d_{\phi_{f}(S)}(z)$ does not contain any integer of $\mathcal{V}_{1} \cup \cdots \cup \mathcal{V}_{k}$. This means in particular that $y$ is a stable state.

Hence, for any state $x$, there exists a stable state $y$ such that $x \rightsquigarrow y$ in $\mathcal{G}_{a}\left(\phi_{f}((S))\right.$, and this is the same for $S$. In conclusion, $\mathcal{G}_{a}(S)$ as no cyclic attractor.

Remark 12. In [8], the negative Thomas'rule is enunciated in the following form:

The presence of a negative circuit of length at least two (somewhere in phase space) is a necessary condition for stable periodicity.

According to this formulation, an alternative boolean statement of this rule should be as follows: "Given $S: X \rightarrow X$ a boolean FDS such that the asynchronous dynamics of $S$ displays a cyclic attractor, then the $R G$ of $S$ contains a negative circuit of length at least two." We avoid "somewhere in phase space" since, as mentioned above, counterexamples show that the existence of a local graph $\mathcal{R} \mathcal{G}_{x}(S)$ including a negative circuit is not necessary.

In our approach, the statement becomes then: "Let $S: X \rightarrow X$ be a boolean FDS such that all the signs of the interactions of the circuits of $\mathcal{R G}(S)$ of length at least two are determined, and all these circuits are positive. Then $\mathcal{G}_{a}(S)$ does not include any cyclic attractor." But in the logical formalism, a self-inhibition creates a flip-flop effect that produces cycles of length two in the asynchronous STG. Moreover, such a cycle can be an attractor, like for instance with three components and the logical rules $\left(g_{1}: \neg g_{1}, g_{2}: \neg g_{1} \wedge g_{3}, g_{3}: g_{2}\right)$, and this is in contradiction with the alternative statement.

\section{Conclusion}

This work explored links between topological features of regulatory graphs and their issued dynamics in the boolean framework. Remark that the framework we use is very general: we made no assumption on the logical functions, and worked within the non-deterministic asynchronous dynamics (although most of the papers consider monotonous functions and synchronous dynamics).

The characterisation of classes of isometric FDS through their common interaction graph features helped in deciphering necessary conditions to generate given dynamical properties. Moreover, it greatly assisted the analytical analysis of generic regulatory motifs. This raised the question of the choice of a representative regulatory graph of a class that gathers dynamics sharing 
same properties. We chose as representative a regulatory graph containing the most positive edges as possible. Indeed, from a mathematical point of view, activations are easier to handle. However, this criteria could be discussed, as it seems that in many biological models, such as in bacteria, inhibitions play a major role in the regulatory mechanisms [10].

Finally, we chose to define classes based on isometries of asynchronous dynamics. This is the maximal requirement of similarity between dynamical properties. We could relax it, for instance considering classes of isomorphic dynamics. We could then address the question of invariants of their regulatory graphs.

\section{References}

[1] T. H. Cormen, C. E. Leiserson, R. L. Rivest, and C. Stein. Introduction to algorithms. MIT Press, 2009.

[2] F. Delaplace. Analogous Dynamics of Boolean Network. arXiv:1411.6135v1, nov 2014.

[3] G. Didier and É. Remy. Relations between gene regulatory networks and cell dynamics in Boolean models. Discrete Applied Mathematics, 160(15), 2012.

[4] A. Garg, A. Di Cara, I. Xenarios, L. Mendoza, and G. De Micheli. Synchronous versus asynchronous modeling of gene regulatory networks. Bioinformatics (Oxford, England), 24(17):1917-25, sep 2008.

[5] L. Glass. Classification of biological networks by their qualitative dynamics. Journal of theoretical biology, 54(1):85-107, oct 1975.

[6] L. Glass. combinatorial aspects of dynamics in biological systems. In Uzi Landman, editor, statistical mechanics ans statistical methods in theory and applications, page 58611. Plenum publishing corporation, 1977.

[7] S. Kauffman. Metabolic stability and epigenesis in randomly constructed genetic nets. Journal of theoretical biology, 22(3):437-467, 1969.

[8] M. Kaufman, C. Soulé, and R. Thomas. A new necessary condition on interaction graphs for multistationarity. Journal of theoretical biology, 248(4):675-85, oct 2007. 
[9] S. Lang. Algebra. Springer edition, 1997.

[10] J. MONOD and F. JACOB. Teleonomic mechanisms in cellular metabolism, growth, and differentiation. Cold Spring Harbor symposia on quantitative biology, 26:389-401, 1961.

[11] B. Mossé and É. Remy. A Combinatorial Exploration of Boolean Dynamics Generated by Isolated and Chorded Circuits. Acta Biotheoretica, 2019 .

[12] É. Remy, B. Mossé, C. Chaouiya, and D. Thieffry. A description of dynamical graphs associated to elementary regulatory circuits. Bioinformatics (Oxford, England), 19(Suppl. 2):172-178, 2003.

[13] É. Remy, B. Mossé, and D. Thieffry. Boolean Dynamics of Compound Regulatory circuits. In A. Rogato, M. Guarracino, and V. Zazzu, editors, Dynamics of Mathematical Models in Biology Bringing Mathematics to Life, pages 43-53. Springer, 2016.

[14] É. Remy and P. Ruet. From minimal signed circuits to the dynamics of Boolean regulatory networks. Bioinformatics, 24(16), 2008.

[15] A. Richard. Negative circuits and sustained oscillations in asynchronous automata networks. Advances in Applied Mathematics, 44(4):378-392, may 2010.

[16] A. Richard and P. Ruet. From kernels in directed graphs to fixed points and negative cycles in Boolean networks. 2013.

[17] F. Robert. Discrete iterations. Berlin Heidelberg New-York, springer-v edition, 1986.

[18] D. Slepian. On the number of symmetry types of Boolean functions of $\mathrm{n}$ variables. Canadian journal of mathematics, 5:135-193, mar 1953.

[19] R. Tarjan. Depth-first search and linear graph algorithms. 12th Annual Symposium on Switching and Automata Theory (swat 1971), 1(2):146$160,1971$.

[20] R. Thomas. On the relation between the logical structure of systems and their ability to generate multiple steady states or sustained oscillations. Springer Series in Synergetics, 9:180-193, 1981.

[21] E. Tonello. Graph properties of biological networks. PhD thesis, Univ. of Nottingham, 2018. 
[22] E. Tonello, E. Farcot, and C. Chaouiya. Local Negative Circuits and Cyclic Attractors in Boolean Networks with at most Five Components. SIAM J. Applied Dynamical Systems, 18(1):68-79, 2019. 